\title{
La flora alóctona de la Comunidad Valenciana (España)
}

\author{
Mario Sanz Elorza ${ }^{1}$, Daniel Guillot Ortiz ${ }^{2}$ Vicente Deltoro ${ }^{3}$
}

Resumen: Sanz Elorza, M.; Guillot Ortiz, D. \& Deltoro, V. 2010. La flora alóctona de la Comunidad Valenciana (España). Bot. Complut. 35: $97-130$.

En este trabajo, continuación de otros anteriores dedicados a la caracterización de la flora alóctona de las diferentes Comunidades Autónomas de España, nos ocupamos de la Comunidad Valenciana. Para ello partimos de la información disponible en la bibliografía, complementada con la de nuestras propias observaciones y experiencia. Los resultados revelan que existen, al menos, 663 especies de plantas vasculares exóticas naturalizadas o subespontáneas en la región, de las cuales el 23\% manifiestan carácter invasor. Dentro de ellas 51 son transformadoras. Por la suavidad de su clima en buena parte del territorio y por la intensa presión humana ejercida sobre el medio, la Comunidad Valenciana presenta una elevada capacidad de acogida de especies de plantas vasculares exóticas que pueden desencadenar episodios de invasión. Así mismo, la administración autonómica valenciana es una de las más avanzadas del estado español, en cuanto a la adopción de medidas legislativas y de gestión dirigidas al control y prevención de las invasiones biológicas.

Palabras clave: plantas vasculares, flora alóctona, invasiones biológicas, Comunidad Valenciana, España.

Abstract: Sanz Elorza, M.; Guillot Ortiz, D. \& Deltoro, V. 2009. The alien flora of Comunidad Valenciana (Spain). Bot. Complutensis. 35: 97-130.

This paper is a continuation of previous ones focused on the alien flora of autonomous regions of Spain. Now, we examine the alien flora of Comunidad Valenciana, an autonomous region of the eastern Spain. The source of data was mainly literature references, complemented with authors' experience. We can stablish, by the results obtained, a regional catalogue of 663 alien vascular plants species, of which $23 \%$ are invasive and 51 taxa are transformers. A number of them are potentially dangerous for natural ecosystems, mainly wetlands and maritime dunes. Invasibility of Comunidad Valenciana by alien plants is higher compared with the close regions due to its mild temperatures and high human presure. Good practice in relation to policies, legislation and management relating to invasive alien species is occuring in Comunidad Valenciana, but it remains scattered in other autonomous regions of Spain.

Key words: vascular plants, alien plants, biological invasions, Comunidad Valenciana, Spain.

\section{INTRODUCCIÓN}

Continuando con la serie de trabajos en los que vamos dando a conocer la flora alóctona de diversas comunidades autónomas del estado español, como han sido los casos de Castilla y León (Sanz Elorza et al. 2008) y de Aragón (Sanz Elorza et al. 2009), abordamos en esta ocasión el estado de la cuestión en la Comunidad Valenciana. A pesar de que hoy en día se dispone de una cantidad de información relativa a las especies exóticas en nuestro país impensable hace apenas unos años, la escasa difusión de la misma sigue pesando como uno de los aspectos más limitantes para las labores tanto del científico como del gestor ambiental. Esta falta de disponibilidad de la informa- ción da lugar que el número de especies invasoras tienda a ser significativamente subestimado (McGeoch et al. 2010). Por tanto, el primer objetivo de este trabajo es compilar toda la información dispersa que existe sobre la presencia de especies de plantas vasculares exóticas en el territorio valenciano, para después abordar su análisis desde variados puntos de vista, tales como la evolución histórica del proceso de introducción de flora alóctona, su incidencia en las diferentes provincias de la comunidad autónoma, los principales atributos de las especies introducidas (taxonomía, origen biogeográfico, tipos biológicos, estatus), la situación en la que se encuentra la Comunidad Valenciana en comparación con otras comunidades autónomas y territorios próximos y el alcance y la suficiencia de

\footnotetext{
${ }^{1}$ Gerencia Territorial del Catastro, C/ Ildefonso Rodríguez 1, E-40001 Segovia, España.mario.sanz@segovia.catastro.meh.es

${ }^{2}$ Fundación Oroibérico, E-44113 Noguera de Albarracín, Teruel, España.dguillot_36@hotmail.com

${ }^{3}$ Consellería de Medio Ambiente, Agua, Urbanismo y Vivienda, Servicio de Conservación y Gestión de la Biodiversidad, C/ Arquitecto Alfaro 39, E-46011 Valencia, España. deltoro_vic@gva.es

Recibido: 30 abril 2010. Aceptado: 26 mayo 2010
} 
las medidas adoptadas hasta la fecha para mitigar los efectos negativos de este fenómeno.

\section{MATERIALES Y MÉTODOS}

Para la elaboración del catálogo de la flora alóctona de la Comunidad Valenciana hemos partido, como es nuestra norma, de la información bibliográfica disponible y del conocimiento y experiencia personales adquiridos a lo largo de nuestro bagaje botánico por la región, especialmente de uno de nosotros. En lo que respecta al criterio nomenclatural seguido se ha priorizado de acuerdo al siguente orden: Flora iberica (Castroviejo et al. 1986-2005), Flora Europaea (Tutin et al. 1964-1980) y Flora of North America (Morin et al. 1993-2007). De igual modo que en los precedentes anteriores, para aquellos taxones no recogidos en las obras mencionadas, o bien indebidamente tratados en ellas, nos hemos atenido a monografías específicas y a floras de ámbito más local. Tal y como recomienda la buena praxis, para reseñar el estatus, invasibilidad o grado de integración de las especies exóticas hemos continuado fieles a las recomendaciones terminológicas de Richardson et al. (2000) y de Pyšek et al. (2004).

\section{RESULTADOS}

La flora alóctona de la Comunidad Valenciana se compone, hasta la fecha, de 663 taxones de nivel específico 0 inferior (Tabla 1). De acuerdo con las estimaciones más recientes de la riqueza florística autóctona (Mateo \& Crespo, 2009), ello supone que aproximadamente $20 \%$ de la flora total ha sido introducida por causas no naturales. Si observamos la evolución histórica del número de especies introducidas (Fig. 1) se detecta un primer punto de inflexión a principios la la década de los cincuenta del siglo $\mathrm{XX}$, habiendo permanecido la pendiente de la curva bastante estable hasta entonces. Aproximadamente 20 años despues la pendiente se dispara adquiriendo la curva una forma exponencial. Se deduce, por tanto, que la situación de la Comunidad Valenciana a este respecto no es muy diferente a la observada en Europa en su conjunto (Pyšek et al.2009), es decir que el aumento de la frecuencia con que se producen introducciones de especies de plantas vasculares exóticas es un fenómeno muy ligado a los tiempos actuales (Dana et al. 2003), aunque en nuestro caso sí que hay diferencias cuantitativas importantes como más adelante veremos. Por provincias, se observan diferencias relativamente acusadas entre Valencia (542 taxones) y Castellón (376 taxones), situándose Alicante en una posición intermedia (429 taxones). La razón de estas diferencias puede radicar en las condiciones orográficas, climáticas y socioeconómicas de Castellón, más montañosa, fría y menos poblada en su interior, que disminuyen su propen- sión a acoger especies de plantas vasculares alóctonas. No vemos en el caso de la Comunidad Valenciana el efecto de sesgos debidos al desigual conocimiento de la flora entre las distintas provincias que pudiera existir, ya que nos encontramos en una de las regiones españolas florísticamente mejor estudiadas, tanto en lo que respecta a la cantidad como a la calidad de los trabajos publicados y también a la abundancia de material depositado en los herbarios.

El análisis del espectro corológico de las especies introducidas muestra que, en general, se encuentra bastante repartido entre los diferentes ámbitos biogeográficos del Mundo. Los mayores porcentajes corresponden a los trópicos (Neotropical 20\%, Paleotropical 9\%) y a América (América del Norte 15\%, América del Sur 5\%), destacando también Asia central ( $9 \%$ ) y oriental (8\%), la región Mediterránea (11\%) y el reino Capense (10\%). Estos resultados se muestran bastante coincidentes con lo observado en otras áreas próximas como Baleares (Moragues $\&$ Rita 2005) y Cataluña (Casasayas 1989), aunque menos con la situación existente en zonas más continentales como Castilla y León (Sanz Elorza et al. 2008) y Aragón (Sanz Elorza et al. 2009), donde el peso de los taxones procedentes de zonas templadas no tropicales es mayor. Si se compara con las regiones costeras atlánticas y cantábricas, como Vizcaya (Campos \& Herrera 2009), Galicia (Romero Buján 2007) y Portugal (Domingues \& Freitas 2001), el contingente de alóctonas mediterráneas y americanas no tropicales es allí superior, si bien lo es menor el de las alóctonas asiáticas, capenses y tropicales s.l., extremos éstos que más adelante discutiremos. La comparación de los orígenes de la flora alóctona valenciana con la de España en su conjunto (Sanz Elorza et al. 2004) muestra un alto grado de semejanza, existiendo ligeras diferencias a favor de las especies capenses y paleotropicales en detrimento de las norteamericanas en el primero de los casos.

La distribución porcentual de los tipos biológicos muestra que los mejor representados dentro de la flora alóctona valenciana son de nuevo los que ocupan los extremos en cuanto a duración de su ciclo biológico, tamaño y grado de lignificación, es decir los terófitos (24\%) y los fanerófitos (37\%). En este sentido, sigue habiendo coincidencia plena con lo observado en otras floras alóctonas peninsulares y mediterráneas. Las vías de introducción son mayoritariamente intencionadas, mostrando la jardinería ( $55 \%$ de los taxones) una superioridad sobre el resto aun más evidente si cabe con respecto al conjunto de España, disminuyendo la proporción de especies introducidas de forma involuntaria (44\%), incluidas las malas hierbas agrícolas (Sanz Elorza et al. 2004). 


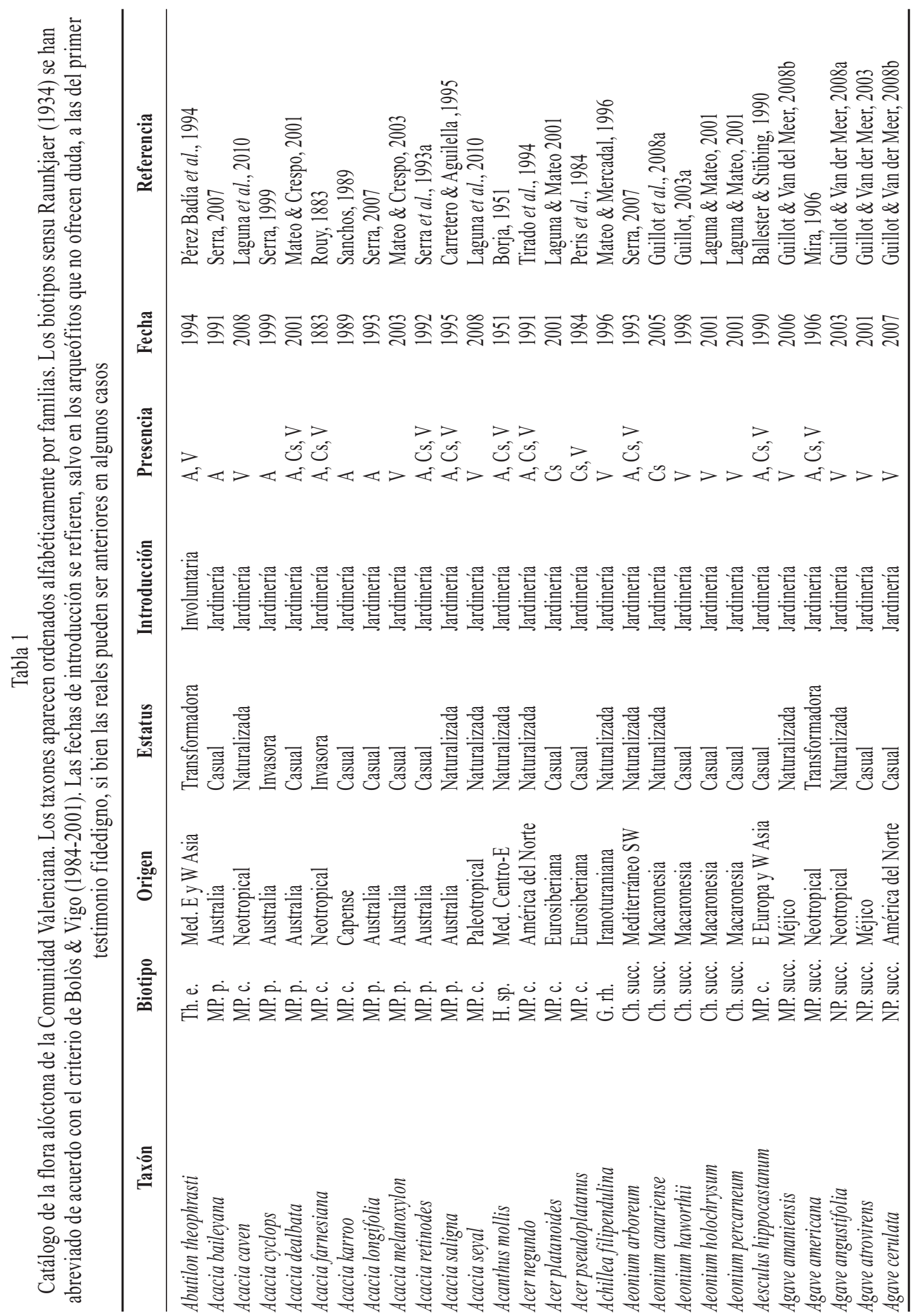




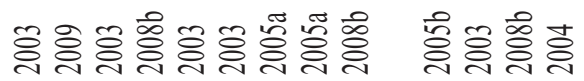

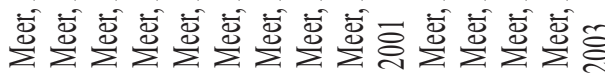

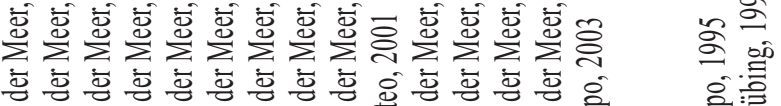

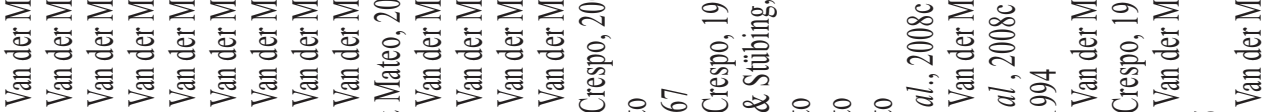

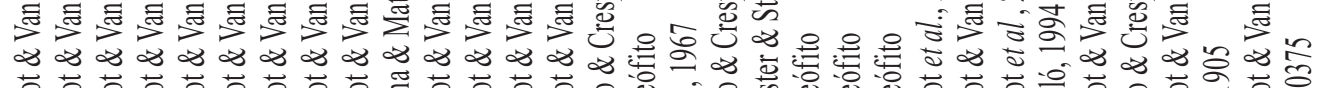

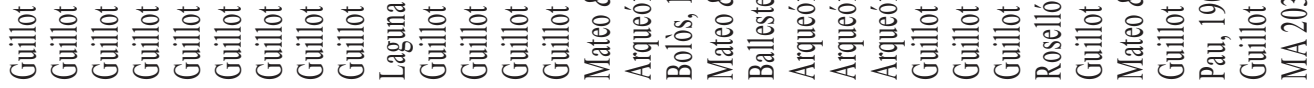

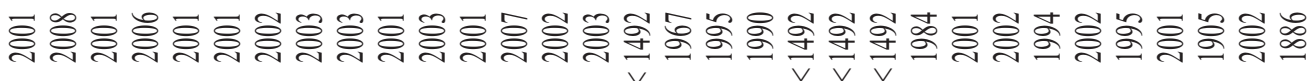

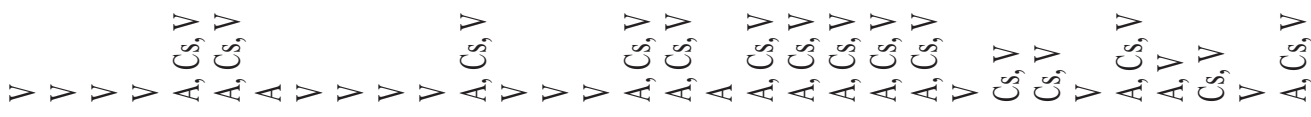

\section{푸}

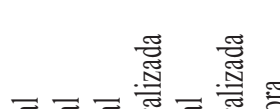

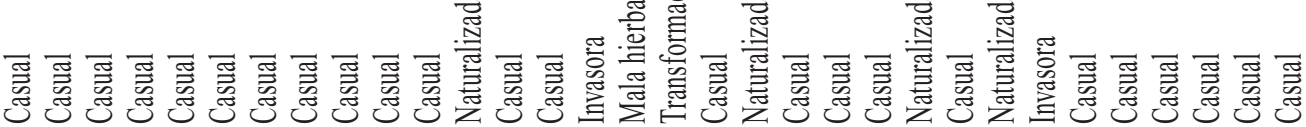

䒕<smiles>c1ccccc1</smiles>

正

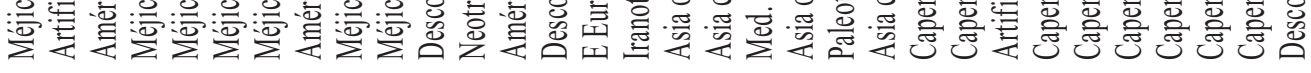

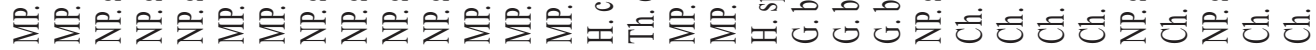

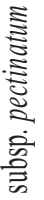

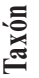

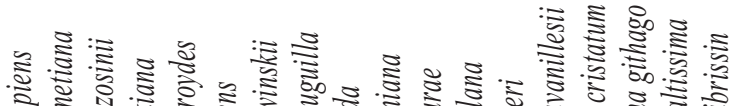

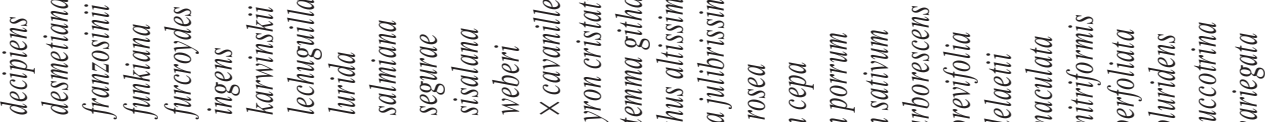

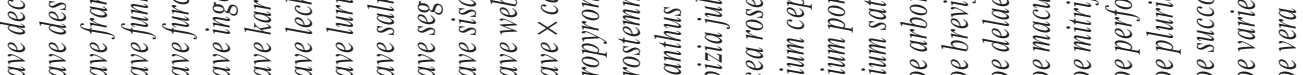
䓌 


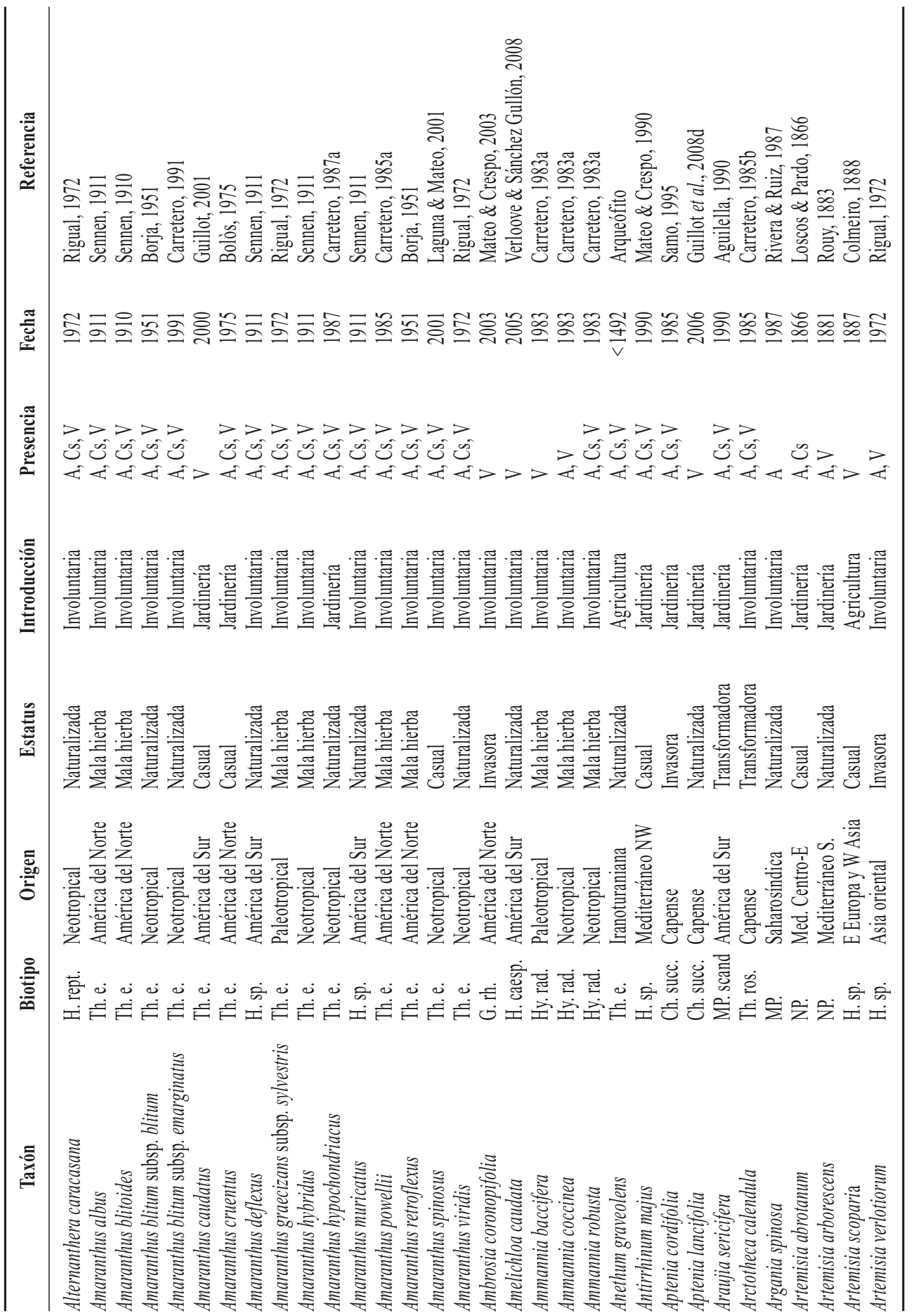




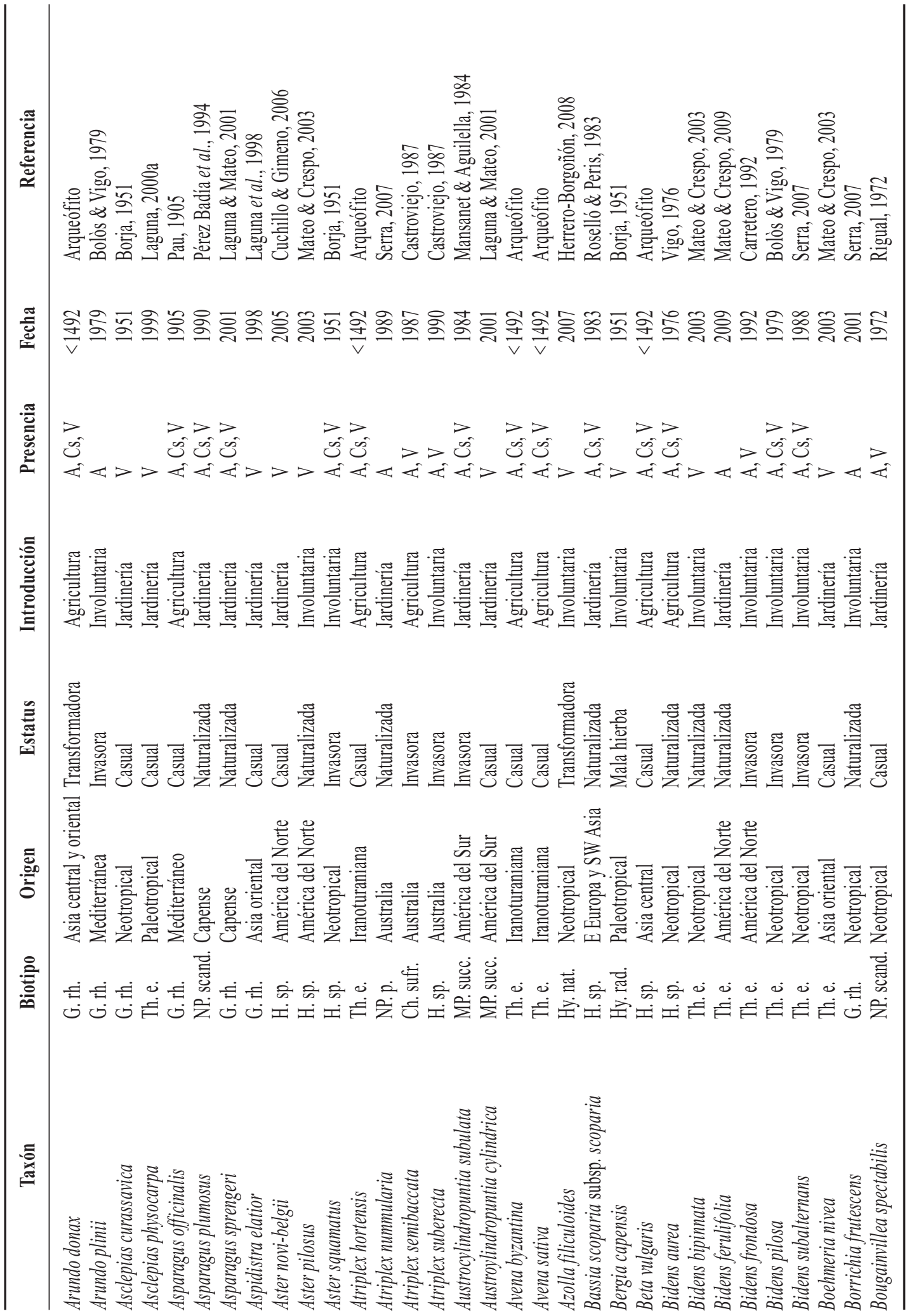




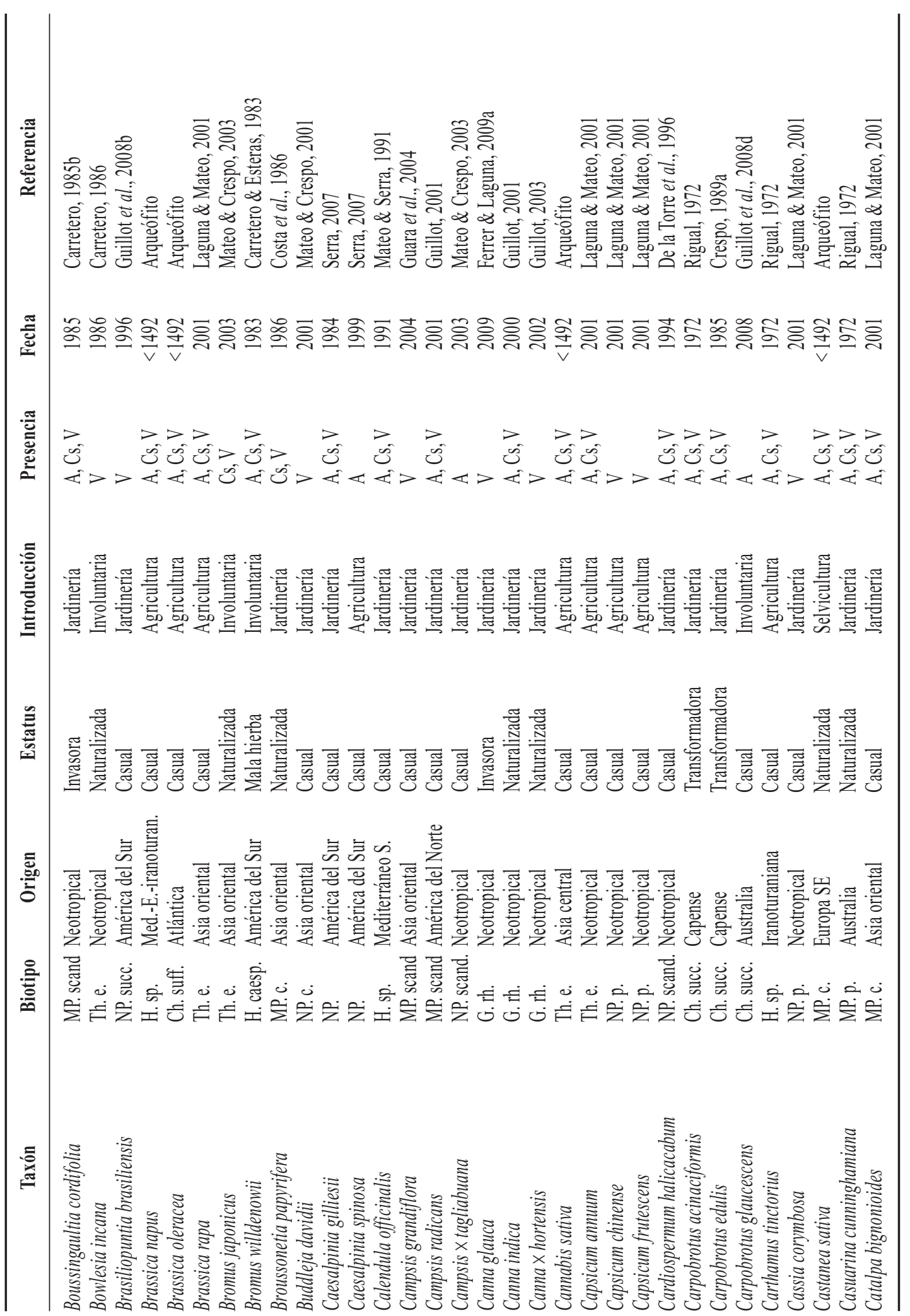




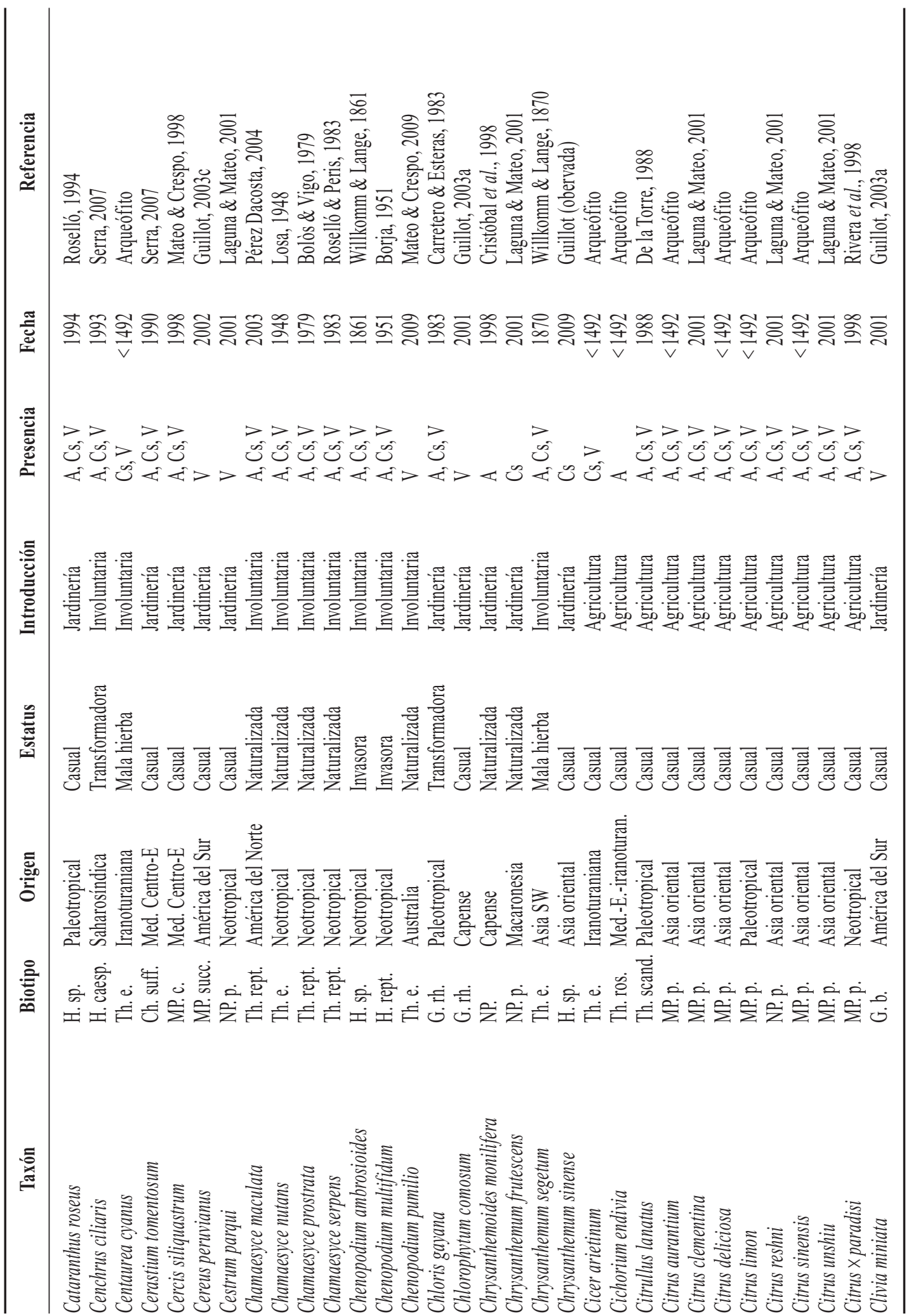




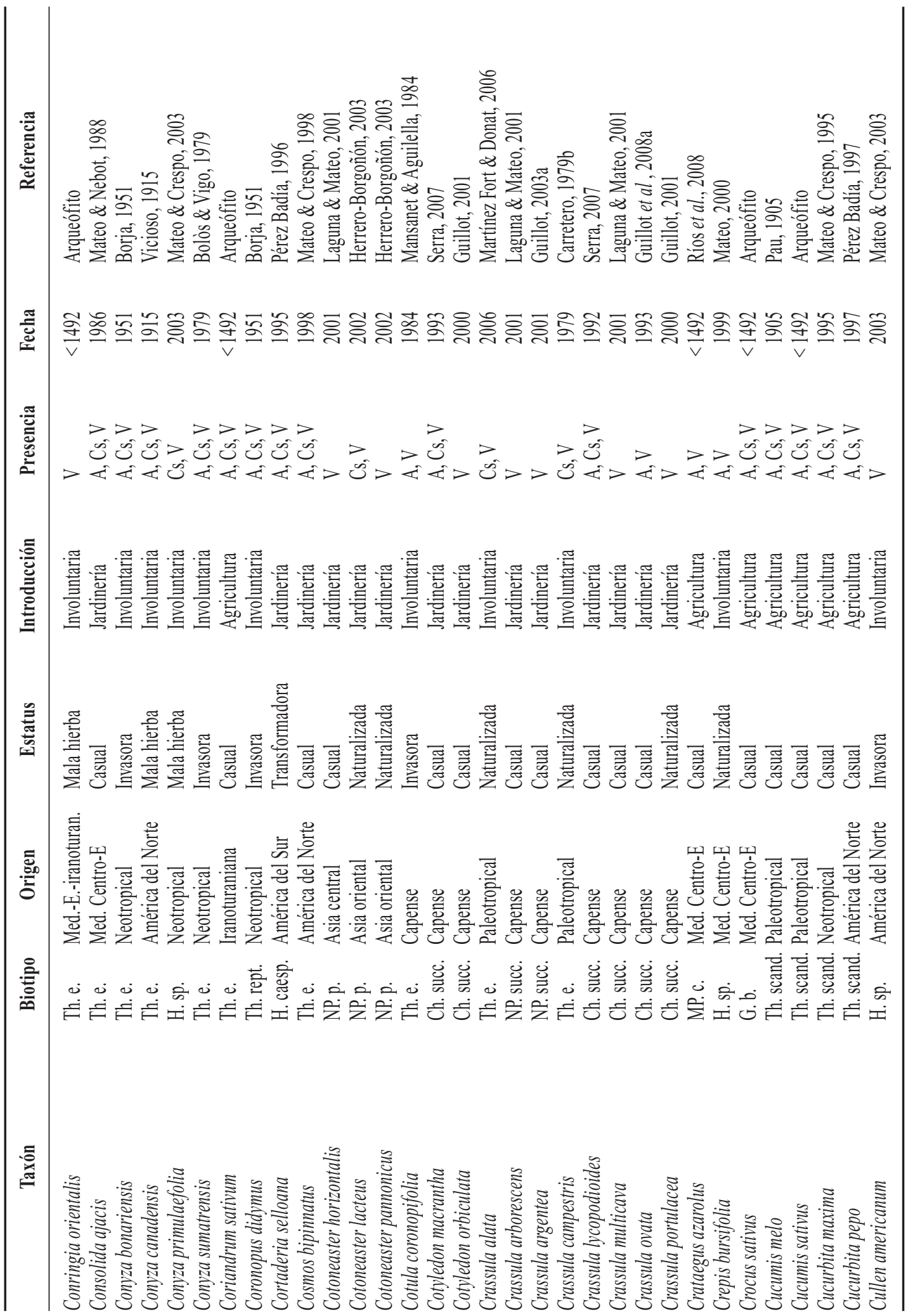




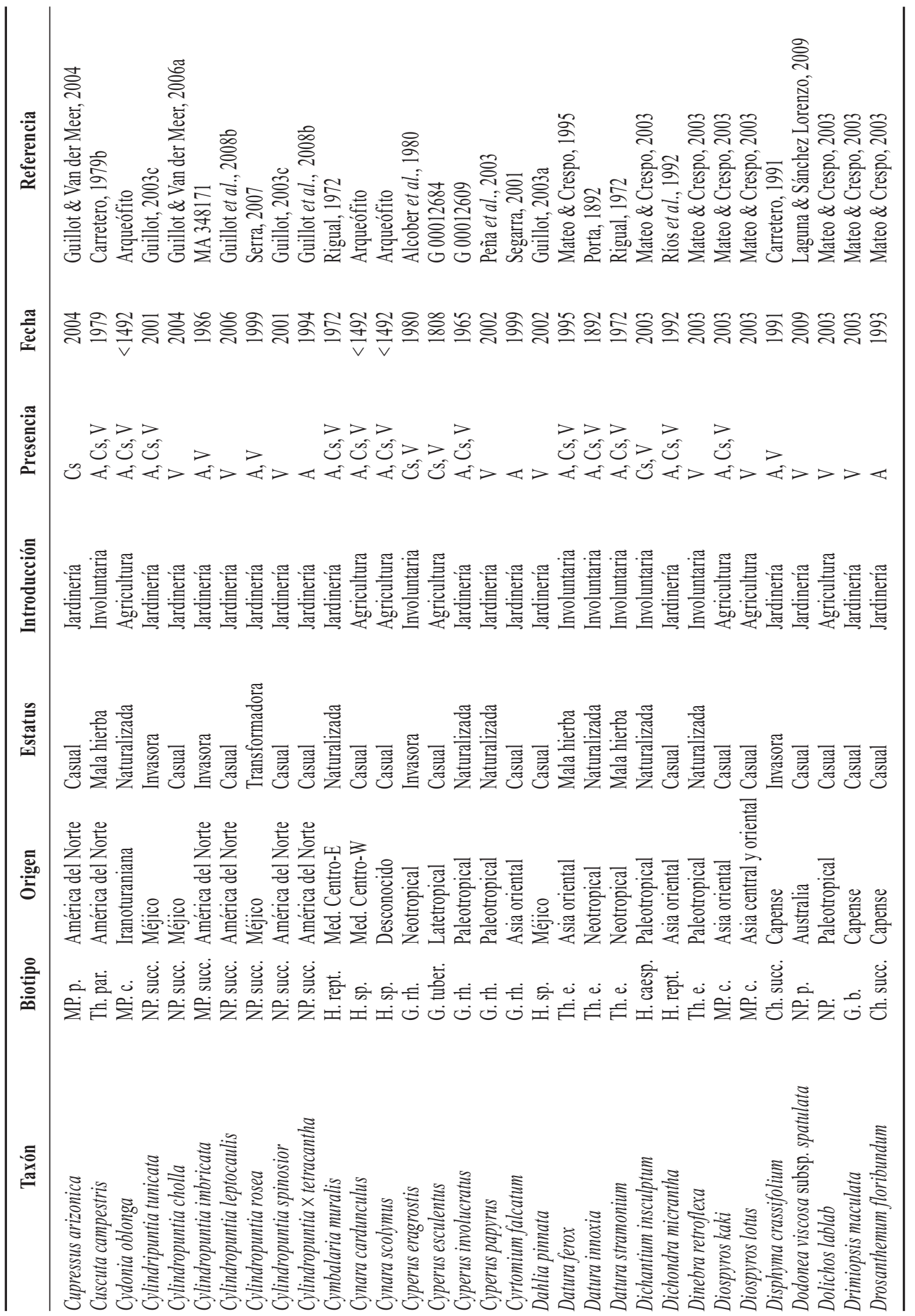




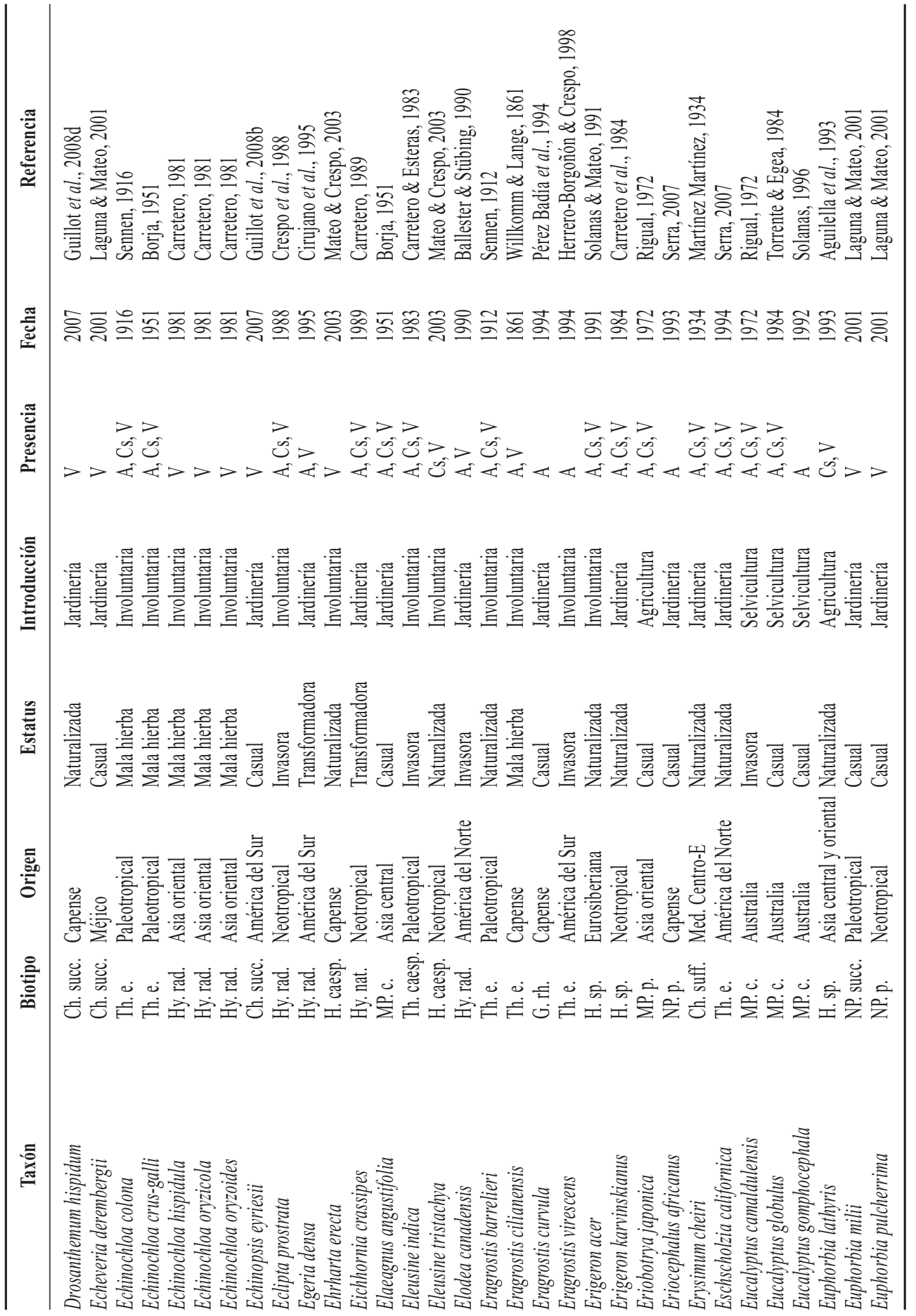




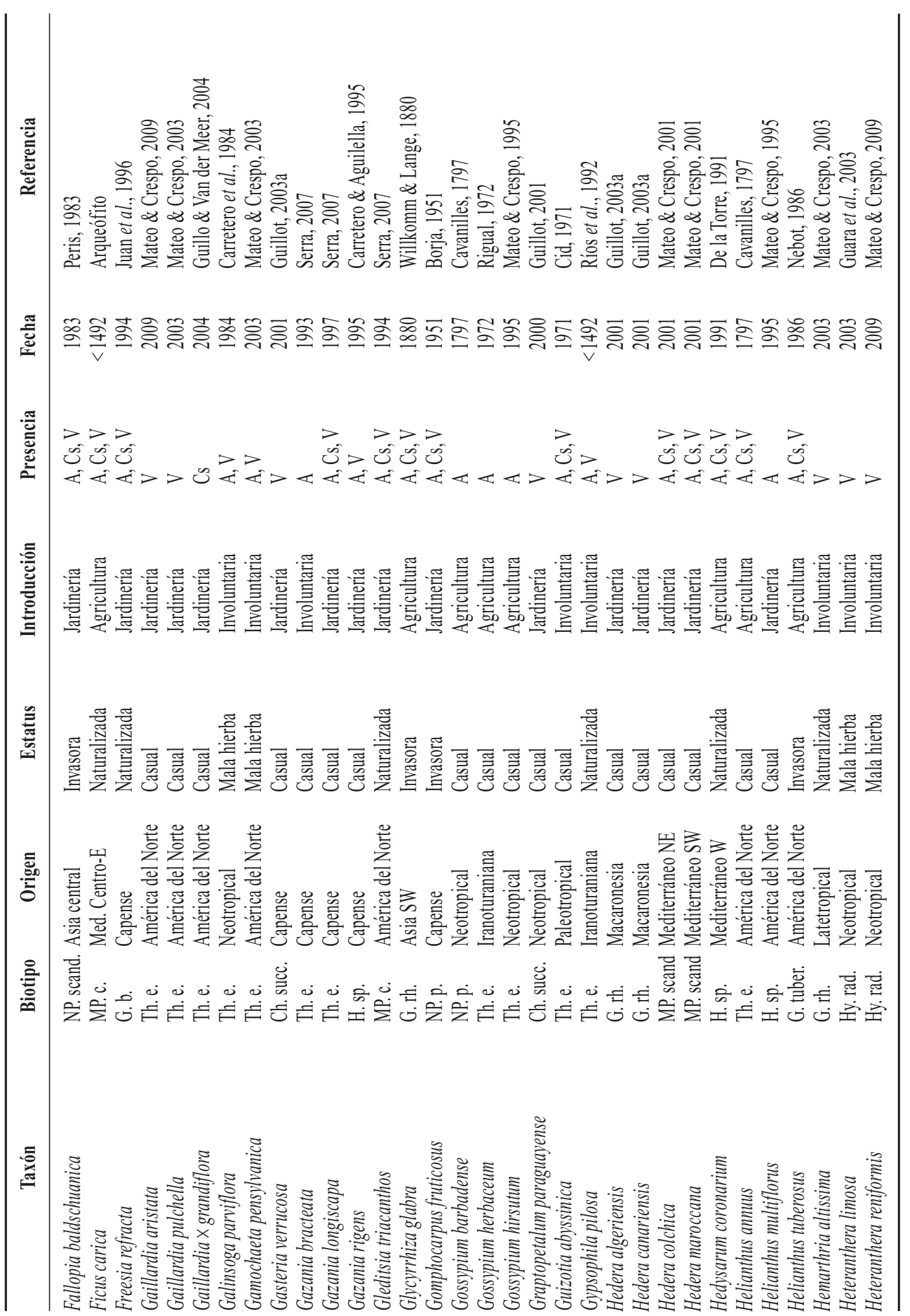




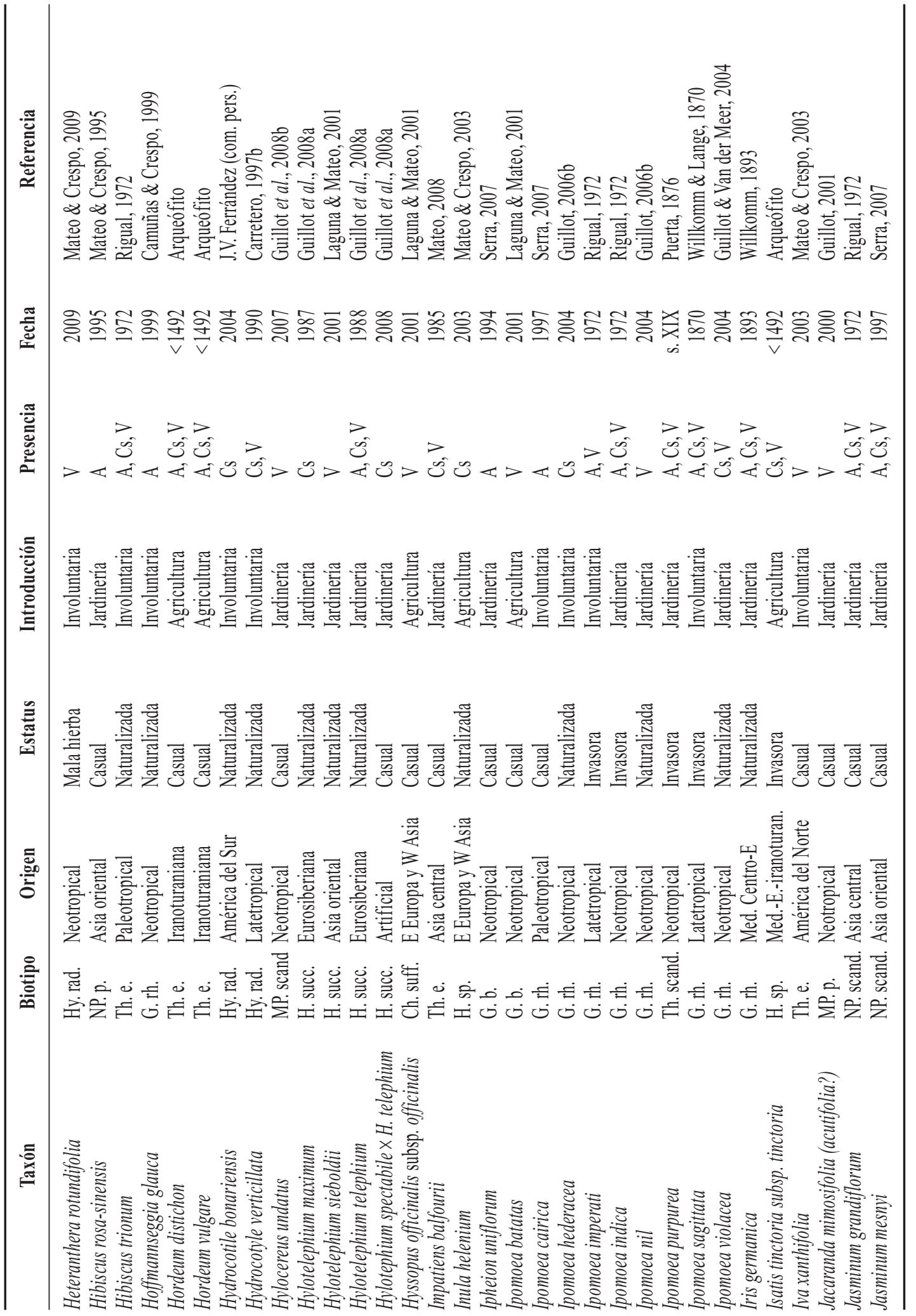




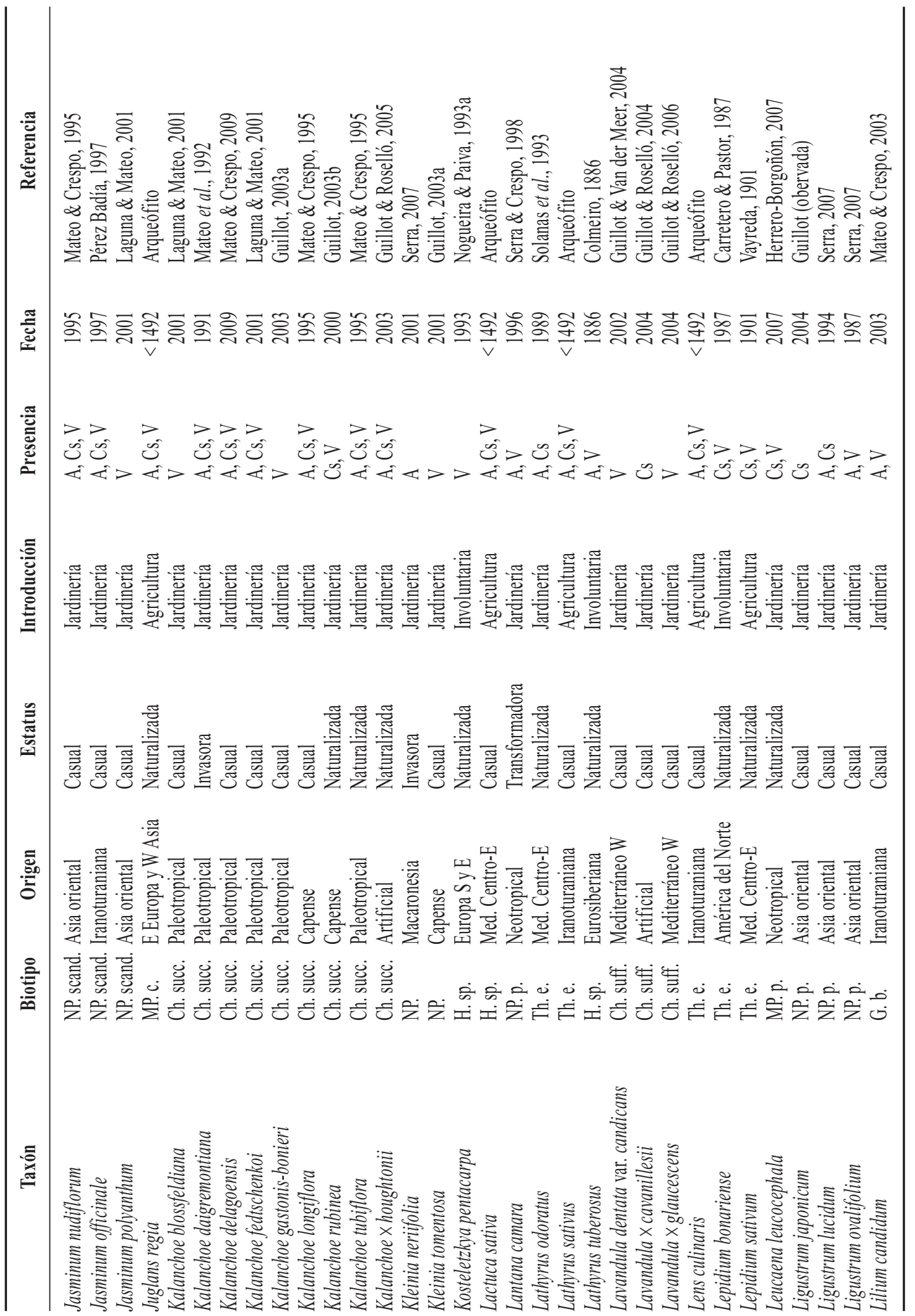




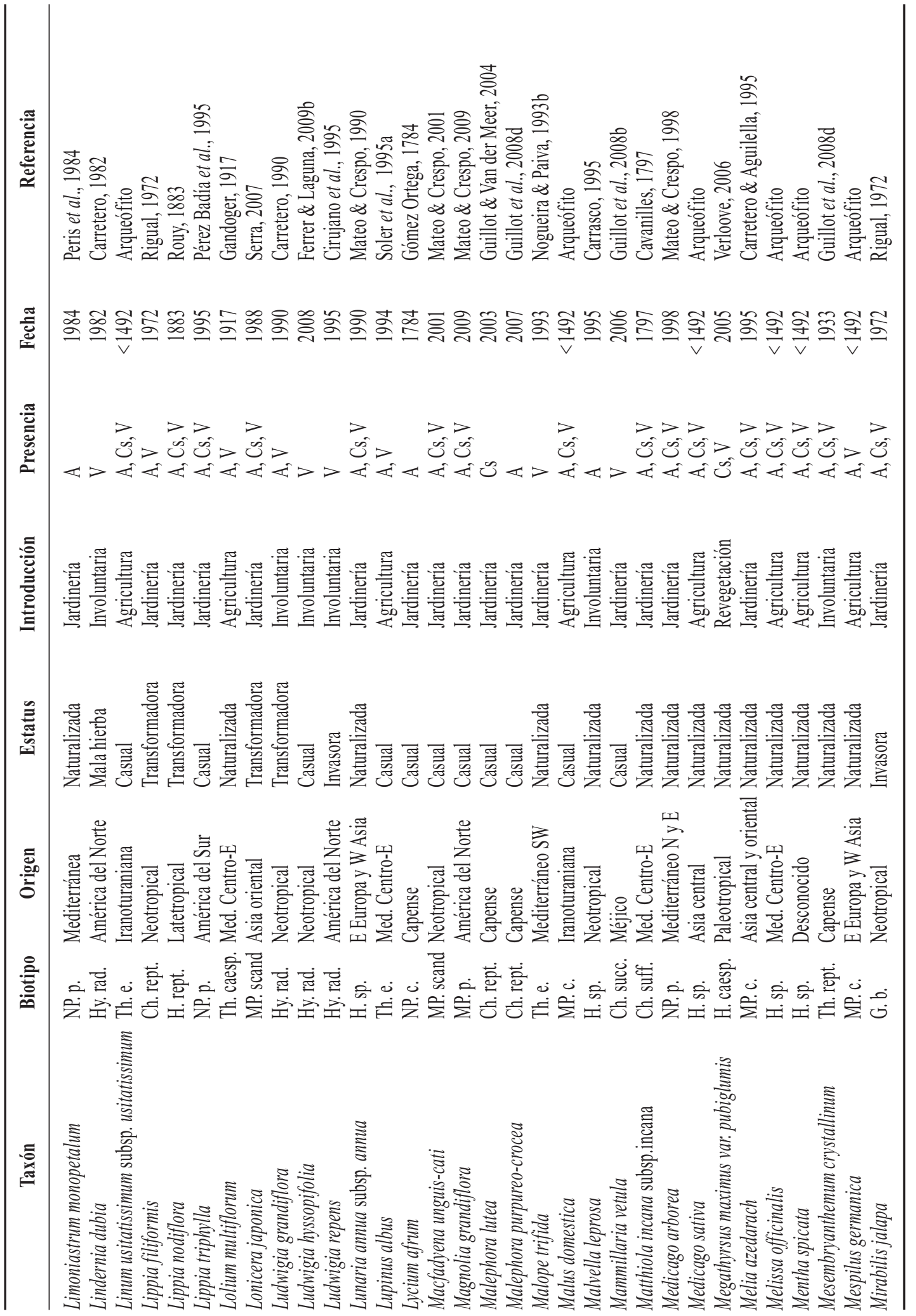




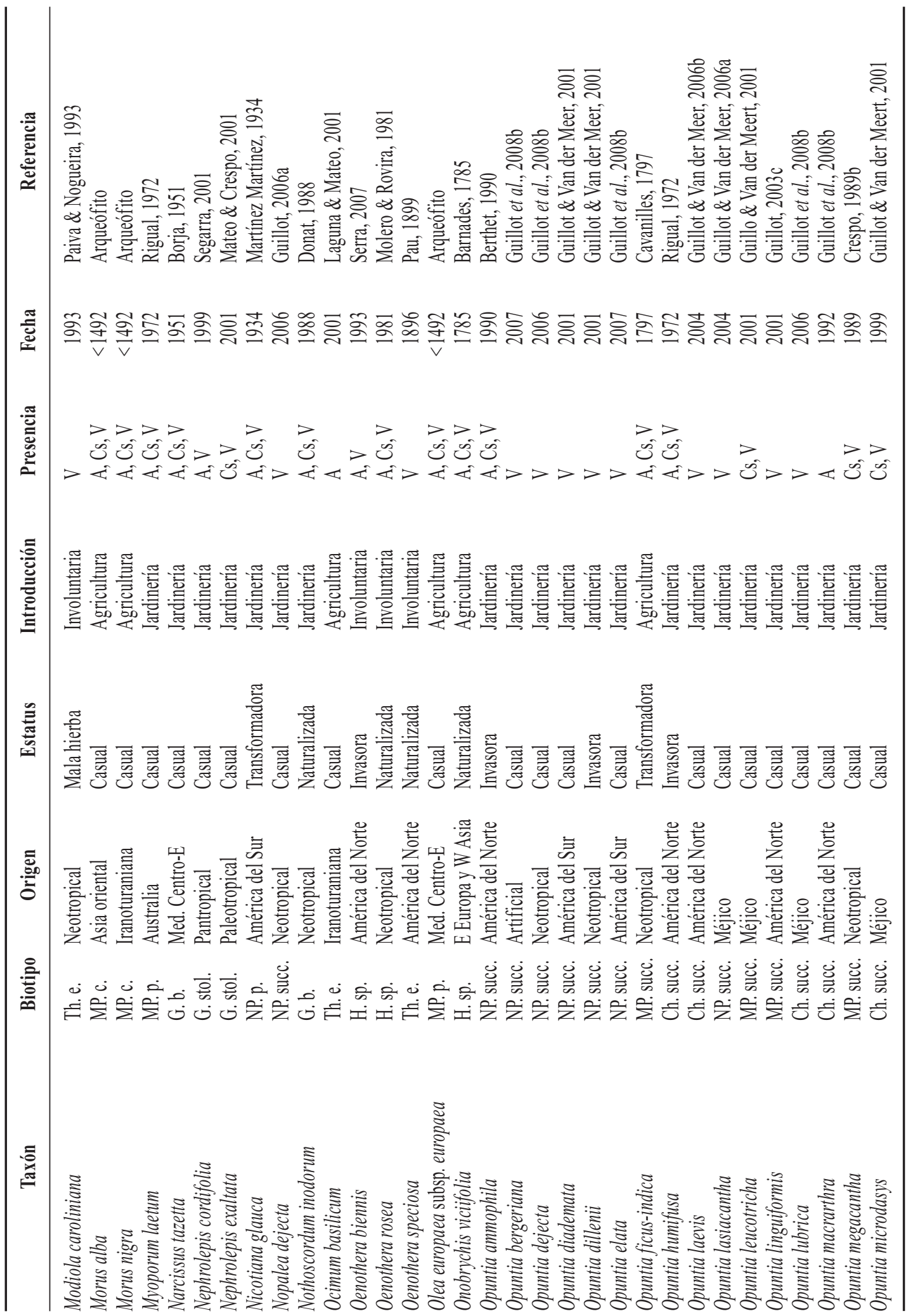




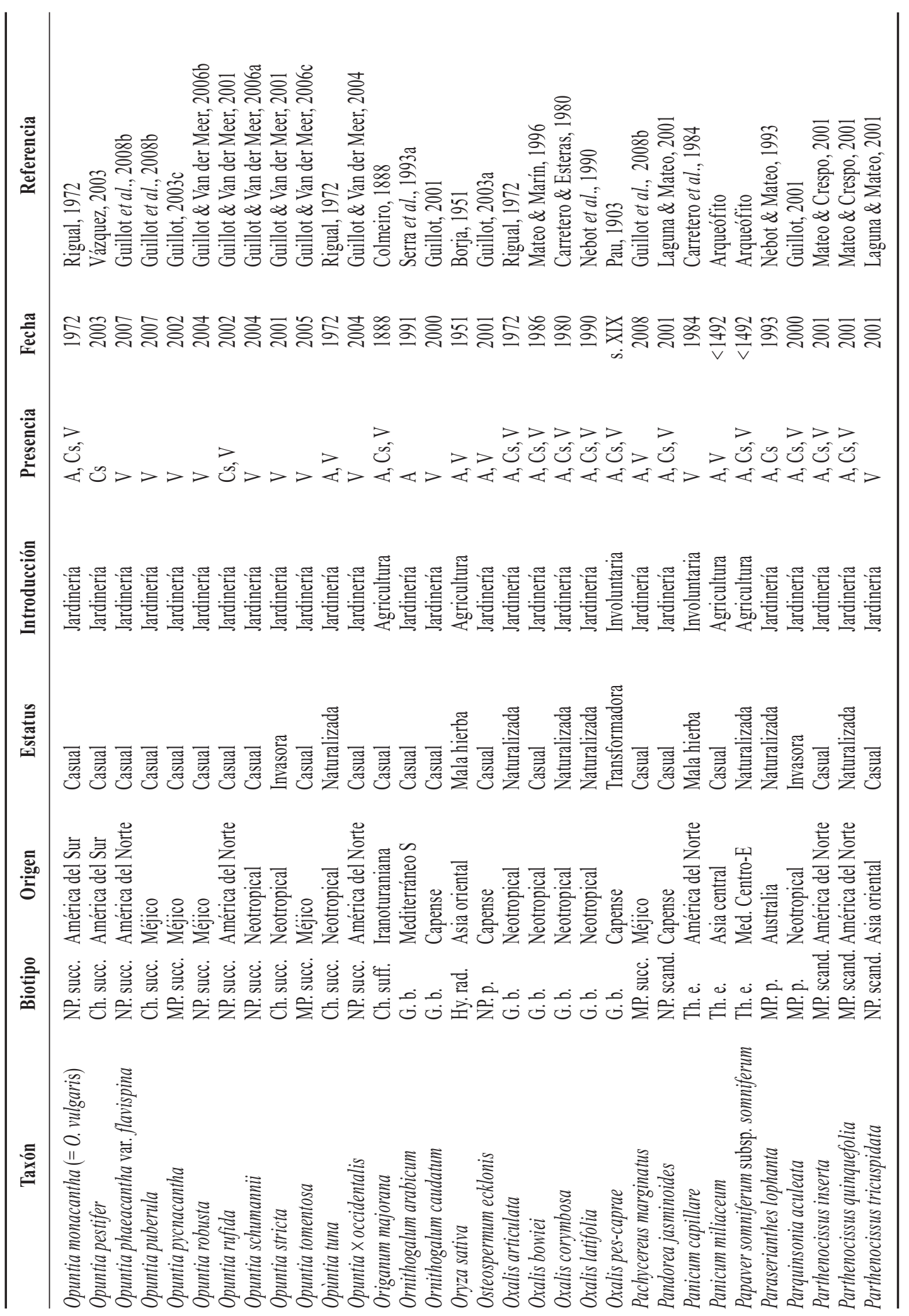




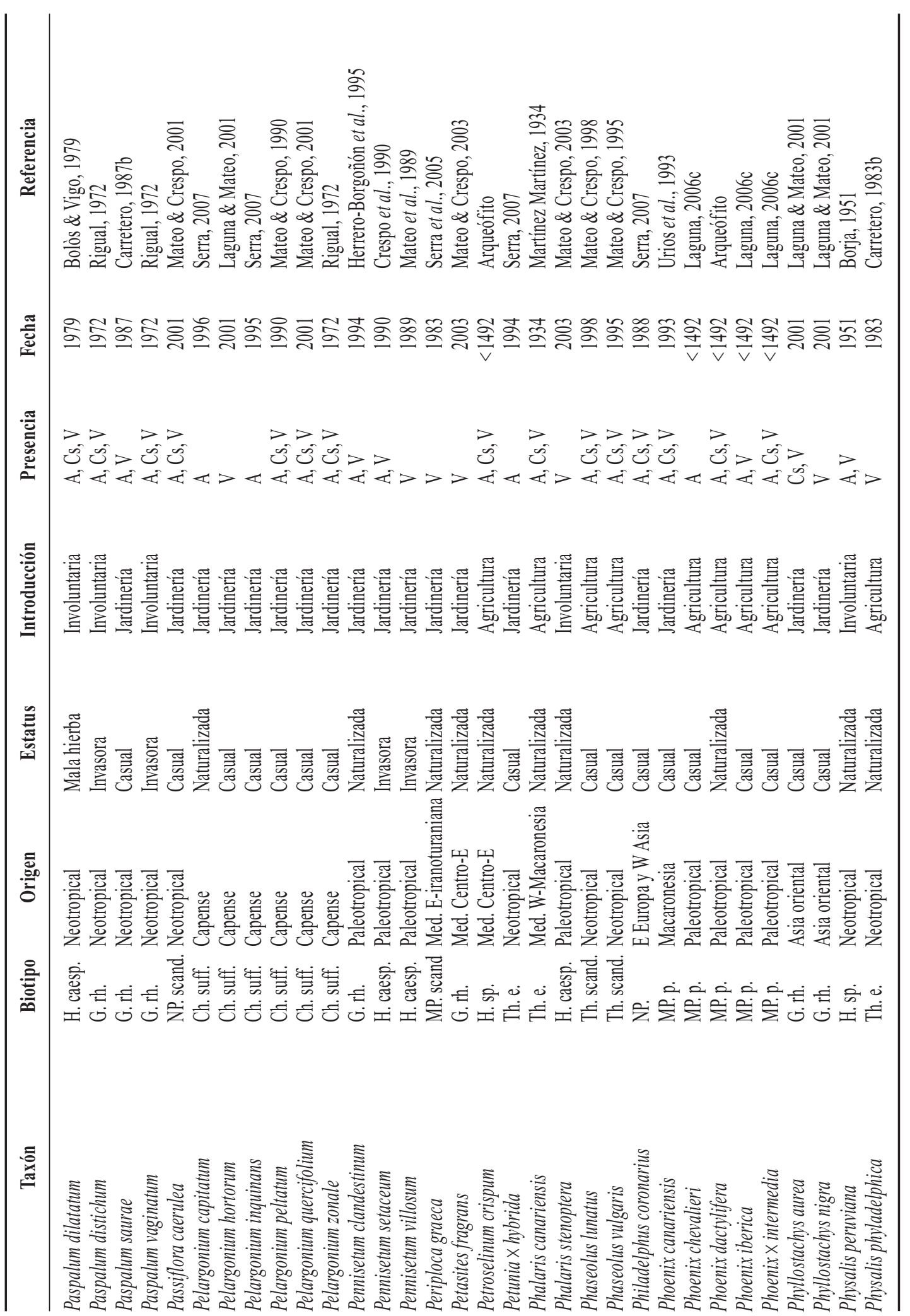




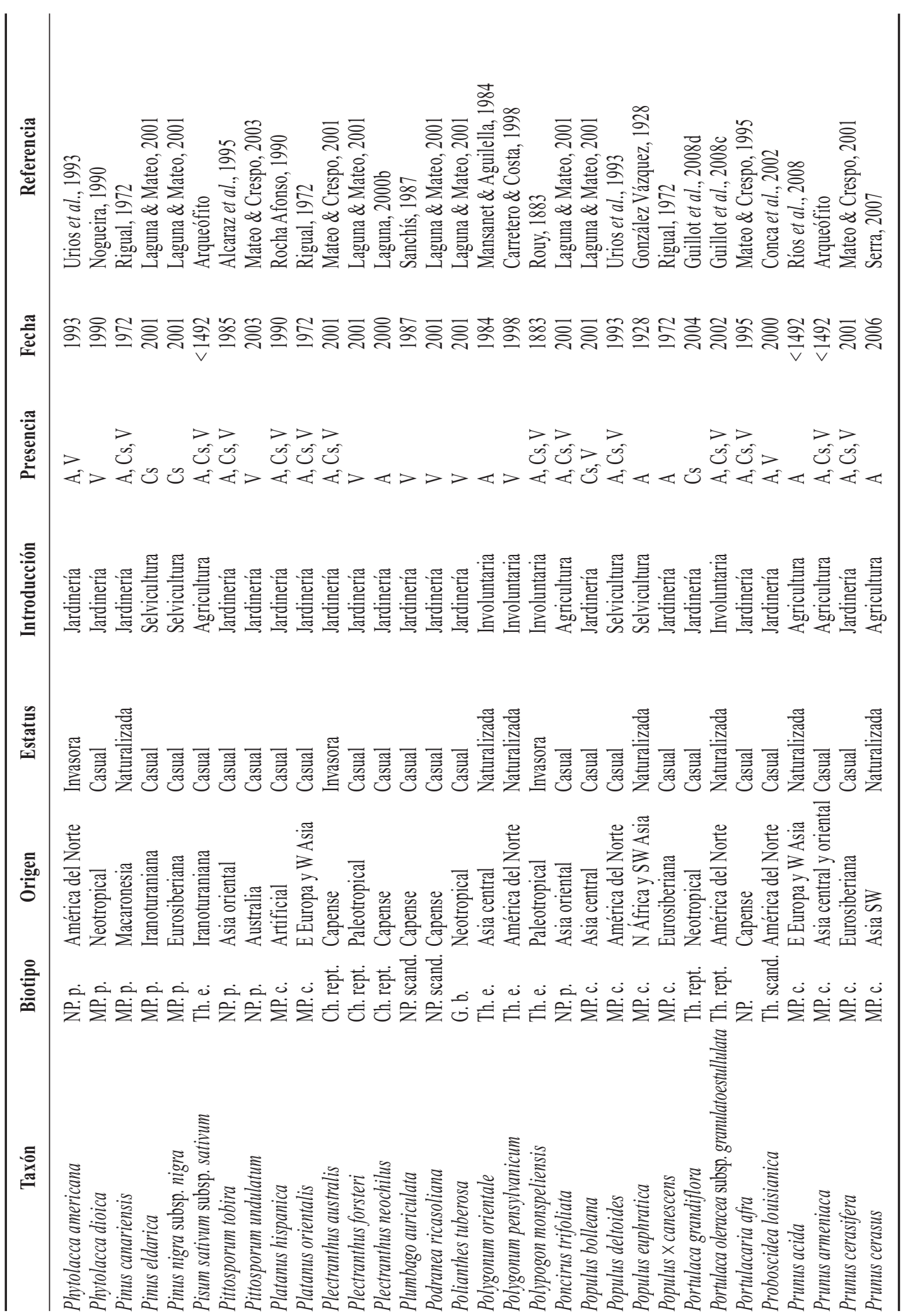




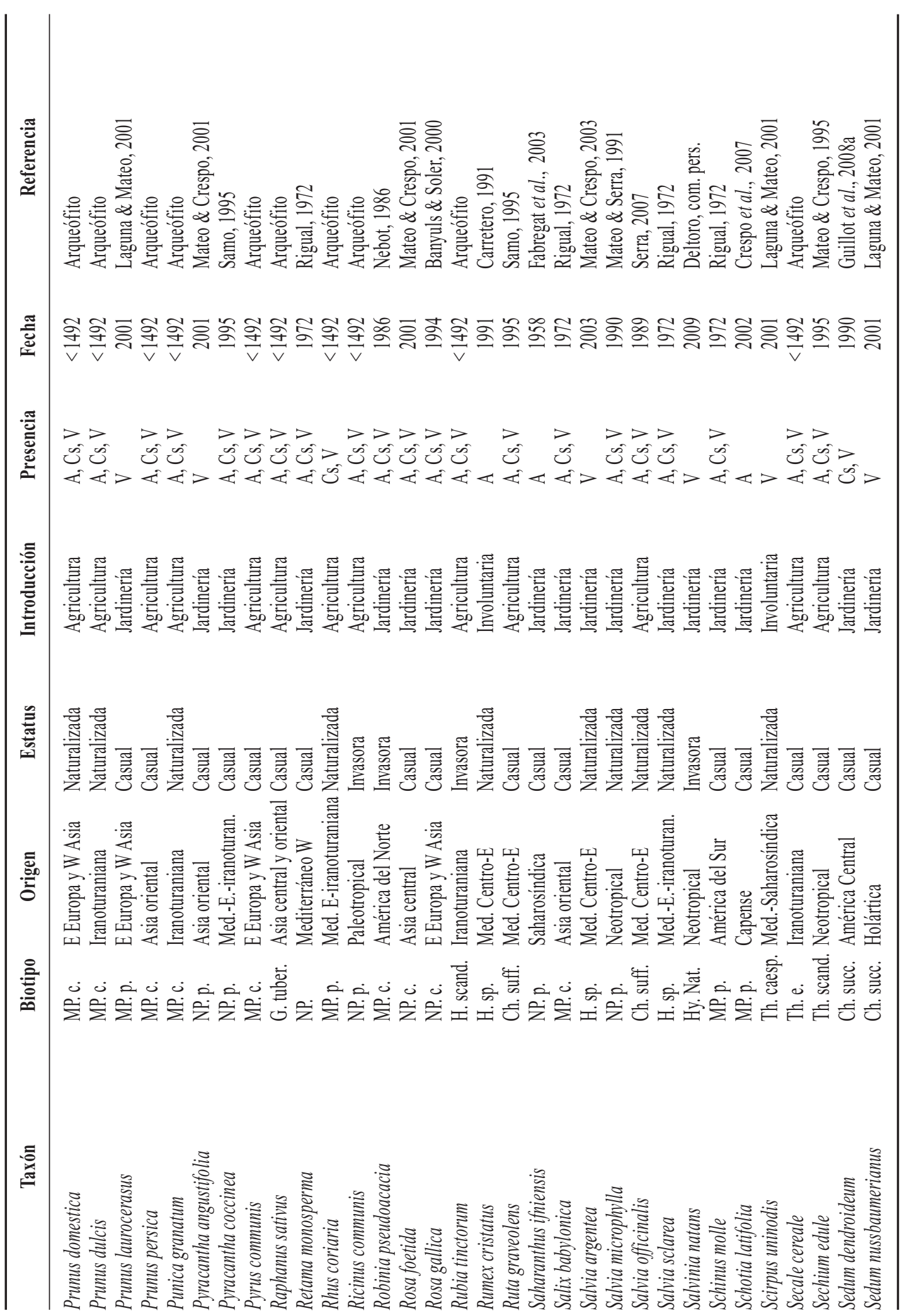




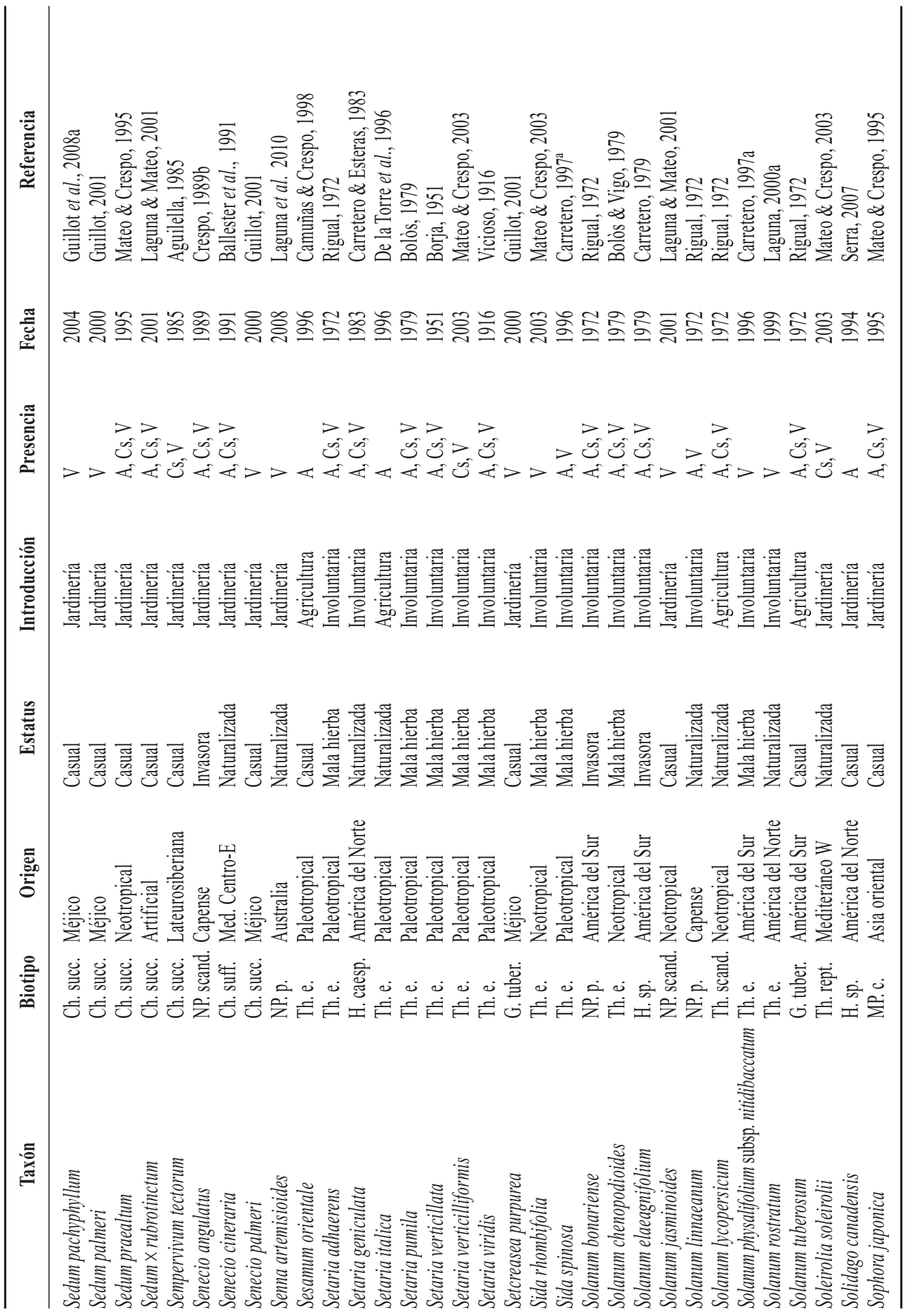




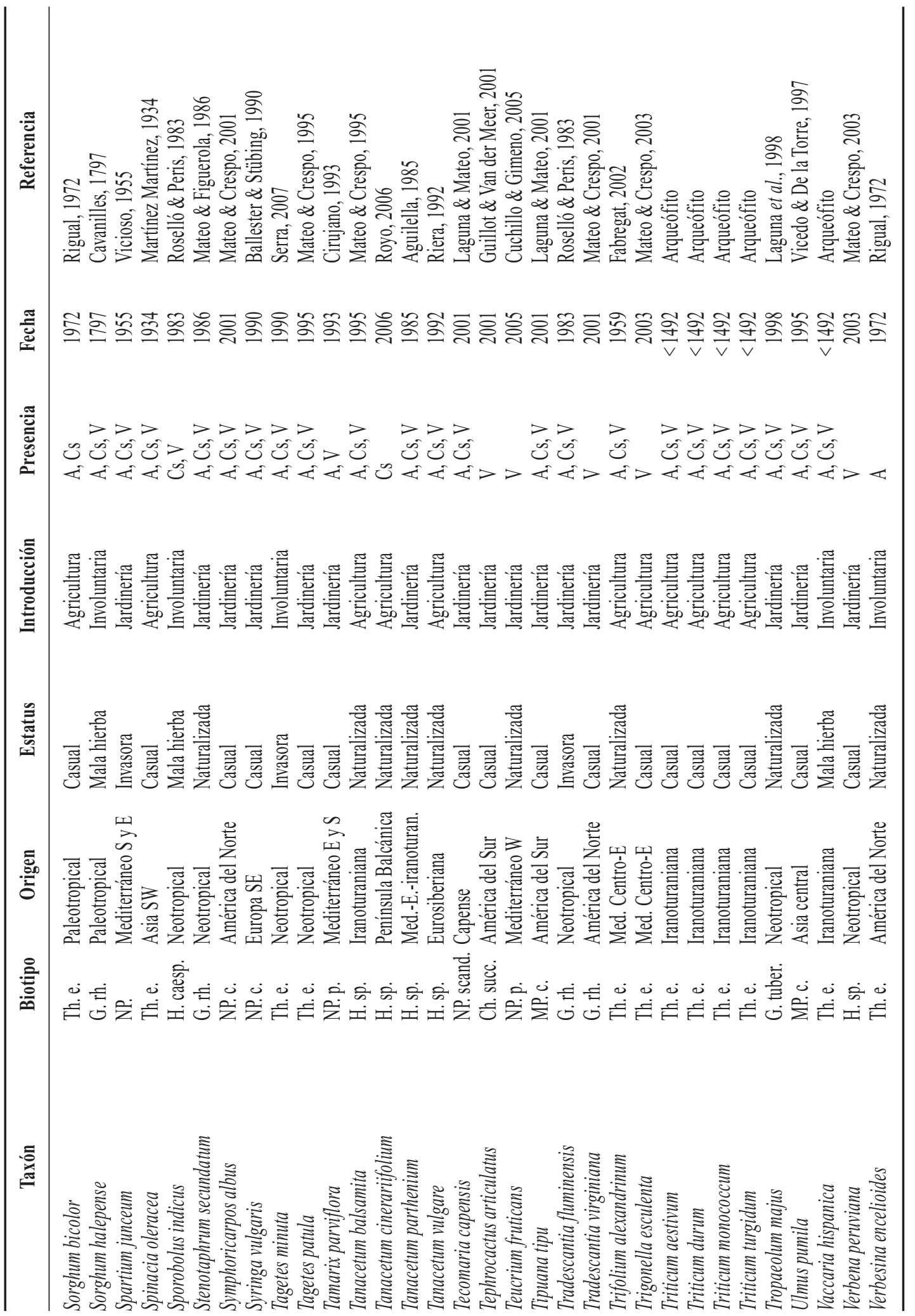




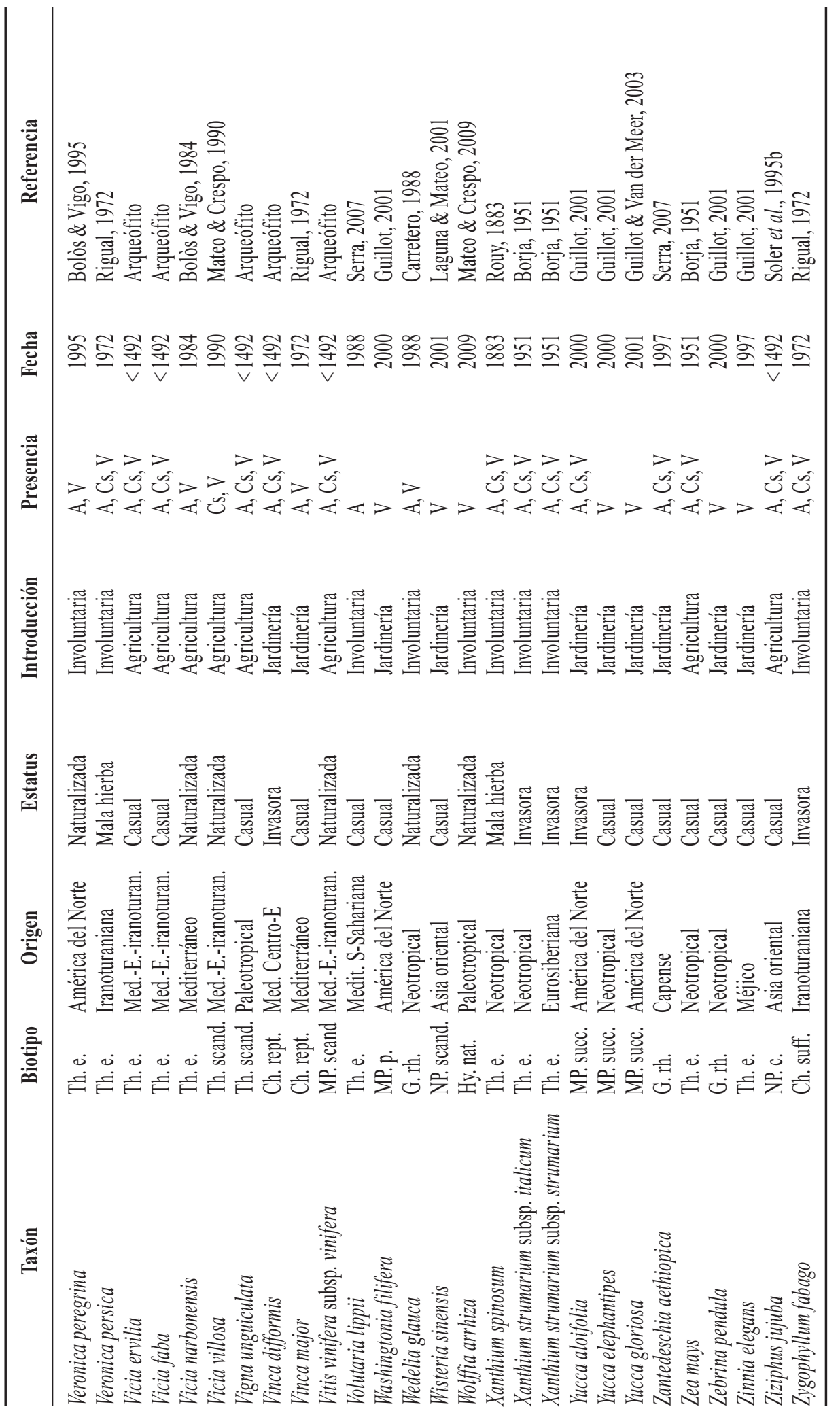




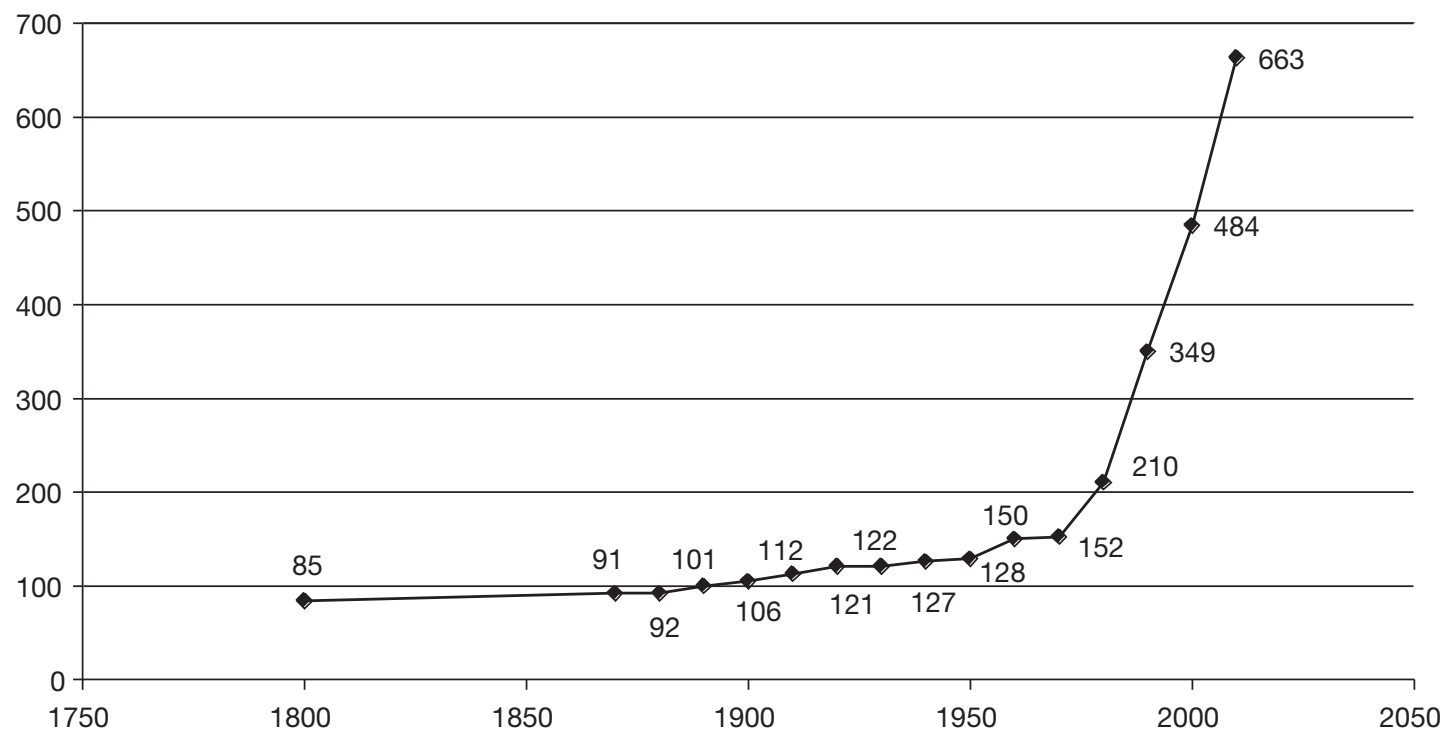

Fig.1- Evolución histórica del número acumulado de especies de plantas vasculares alóctonas introducidas en la Comunidad Valenciana, en los últimos 200 años.

En cuanto al grado de naturalización de la flora alóctona de la Comunidad Valenciana y su carácter invasor (Fig. 2), algo más de la mitad de las especies que la integran son casuales y sólamente el $3 \%$ puede decirse que son transformadoras, es decir con capacidad demostrada para modificar ambientes y ecosistemas. La densidad de xenófitas de la comunidad valenciana (Fig. 3) es la mayor de las observadas hasta ahora en las comunidades autónomas peninsulares, solo superada por las de la cornisa cantábrica (País Vasco y Asturias) y, por supuesto, por los archipiélagos. Estos últimos datos ponen de manifiesto, una vez más, la mayor capacidad de acogida de especies de plantas vasculares alóctonas que presentan las zonas costeras e insulares con respecto a las continentales (Sobrino et al. 2002; Sanz Elorza et al. 2006).

Con relación al análisis taxonómico de la flora alóctona valenciana, podemos decir que se ecuentran representadas 96 familias, encabezadas por Compositae, Gra-

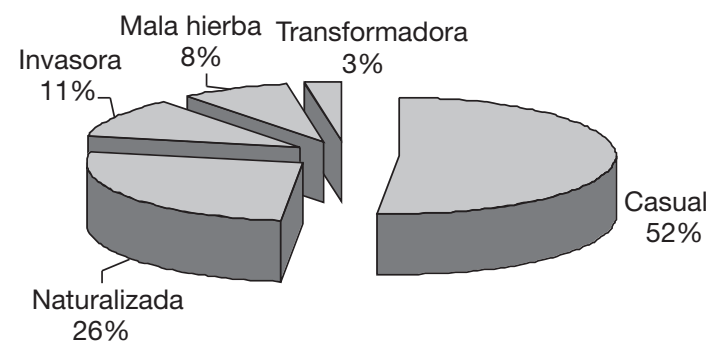

Fig. 2- Distribución porcentual del estatus (grado de integración). mineae y Leguminosae, lo que está en consonancia con el resto de las floras alóctonas de nuestro entorno y con la importancia de estas tres familias en el conjunto del Reino Vegetal. Sí que resulta singular para el caso valenciano la presencia sobrerrepresentada de las familias Cactaceae, Crassulaceae, Agavaceae, Aizoaceae y Aloaceae, cuyas circunstancias y vicisitudes analizaremos en el apartado siguiente.

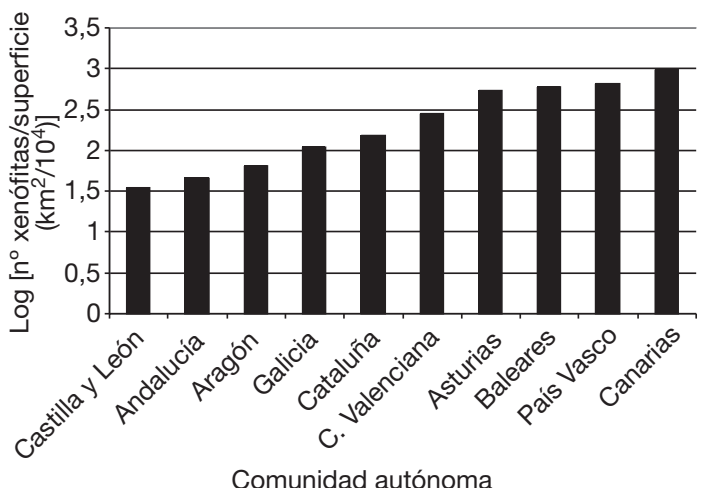

Fig. 3- Densidad de especies de plantas vasculares alóctonas en diversas comunidades autónomas del estado español. Procedencia de los datos: Castilla y León (Sanz Elorza et al. 2008), Andalucía (Dana et al. 2005), Aragón (Sanz Elorza et al. 2009), Galicia (Romero Buján 2007), Cataluña (Casasayas 1989), Asturias (Cires et al. 2006), País Vasco (Campos \& Herrera 2009), Baleares (Moragues \& Rita 2005), Canarias (López et al. 2003). 


\section{DISCUSIÓN}

El número total de especies de plantas alóctonas presentes en un espacio de la amplitud de la Comunidad Valenciana cabe considerarlo alto, si lo comparamos con los resultados obtenidos en territorios próximos (Aragón, Castilla y León, etc.). Esto es debido a la coincidencia de factores que facilitan el asentamiento y la aclimatación de las mismas, lo que no ocurre en los otros territorios aludidos. En buena parte del ámbito espacial valenciano convergen, por un lado, unas condiciones térmicas atenuadas por el efecto tampón del mar, la baja altitud y la propia situación geográfica, sin fuertes contrastes estacionales y con temperaturas invernales suaves, y un régimen pluviométrico más bien escaso e irregular aunque en cierta medida compensado por el amplio desarrollo del regadío. Por otra parte, la estructura demográfica de la región, con una elevada densidad de población humana en las áreas costeras ha traido consigo una intensa urbanización del territorio, a veces muy alejada de la sostenibilidad. Tampoco hay que perder de vista el efecto del tipo de agricultura practicado, muy intensivo y con fuerte arraigo y larga tradición, que ha causado una intensa artificialización de los agrosistemas y ha abierto una vía de entrada fácil para numerosas especies exóticas arvenses, nitrófilas e higronitrófilas. Todas ellas son circunstancias que favorecen la creación de hábitats con alto grado de invasibilidad y la existencia de una elevada presión de propágulos de especies exóticas creada por la propia actividad humana (jardinería, agricultura, transporte, etc.).

A la hora de interpretar la Fig. 1, hay que tener en cuenta que lo que se toma en consideración es la fecha de la primera evidencia científica (cita bibliográfica, pliego de herbario, etc.) que delata la presencia de cada especie, lo que a veces no coincide con la de su introducción, que puede ser bastante anterior.

Las diferencias en los orígenes predominantes de la flora alóctona que se observan entre comunidades autónomas, tienen relación con factores bioclimáticos y biogeográficos (Sobrino et al. 2002). En efecto, las áreas con clima más genuinamente mediterráneo y con muchos kilómetros de costa (Comunidad Valenciana, Baleares, Cataluña) acogen un mayor número de especies procedentes de zonas tropicales y subtropicales, mientras en Aragón, Castilla y León, Galicia o el País Vasco, dominan las originarias de áreas templadas.

La mayor proporción de terófitos y de fanerófitos observada en el análisis de los tipos biológicos es una característica común a las xenofloras mediterráneas. En tales condiciones resultan más competitivas las especies de ci- clo anual corto, capaces de adaptarse a las diferencias estacionales de temperatura y a la irregular disponibilidad hídrica, tal y como hacen las especies mediterráneas autóctonas (Clary 2008). Por otra parte, la abundancia relativa de especies leñosas tiene su explicación en el origen ornamental de muchas de ellas, pues a través de la jardinería se ha introducido un número mayor de árboles y arbustos que de plantas herbáceas (Sanz-Elorza et al. 2004), más aun tratándose de una región donde la jardinería ha alcanzado un desarrollo inusitado debido al modelo de crecimiento urbano expansivo desarrollado en las últimas décadas, donde han proliferado las urbanizaciones de segundas residencias cuya jardinería no se ha diseñado atendiendo precisamente a criterios de sostenibilidad en lo que respecta al uso de especies exóticas potencialmente invasoras ni de eficiencia en la utilización de los recursos hídricos. Con respecto a este último apunte, resulta mucho más adecuado emplear especies mediterráneas autóctonas (Fraga 2009) en lugar de acudir a xerófitas exóticas, como se ha venido haciendo, y que es el motivo por el que aparecen tantos taxones pertenecientes a las familias Cactaceae, Aizoaceae, Agavaceae, Aloaceae y Crassulaceae, entre los que se encuentran algunas de las especies invasoras más nocivas en nuestro ámbito cercano (Carpobrotus edulis, Carpobrotus acinaciformis, Cylindropuntia rosea, Agave americana, etc.).

Finalmente, y enlazando con lo anterior, hay que dedicar un comentario aparte a la flora invasora, que en la Comunidad Valenciana supone el 22\% de la xenoflora, entre especies simplemente invasoras, transformadoras y malas hierbas. Dentro de ellas, es menor el porcentaje que suponen las malas hierbas (8\%) en el sentido agrícola del término, contrastando este dato con lo observado en las regiones continentales de la Península Ibérica, como es el caso de Castilla y León (Sanz-Elorza et al. 2008) y Aragón (Sanz-Elorza et al. 2009), pero coincidiendo con lo que acontece en las regiones costeras, como Asturias (De la Torre 2003) o el litoral de Cataluña (Sobrino et al. 2002), donde el papel de la agricultura, a pesar de su intensificación, se encuentra eclipsado frente al de la jardinería, que es la que aporta la mayor parte del contingente de especies exóticas, y dentro de éstas las invasoras (Sanz-Elorza et al. 2009). Con respecto al término mala hierba, que a algunos botánicos les resulta incómodo por considerarlo demasiado despectivo, coincidimos con Fernández Quintanilla (2009) que fundamenta la validez de su utilización basándose en los criterios de la Academia Española de la Lengua, que considera que un término es el más adecuado cuando se puede demostrar que su uso es también el más habitual. Tan sólo el 3\% (20 taxones) de la flora alóctona 


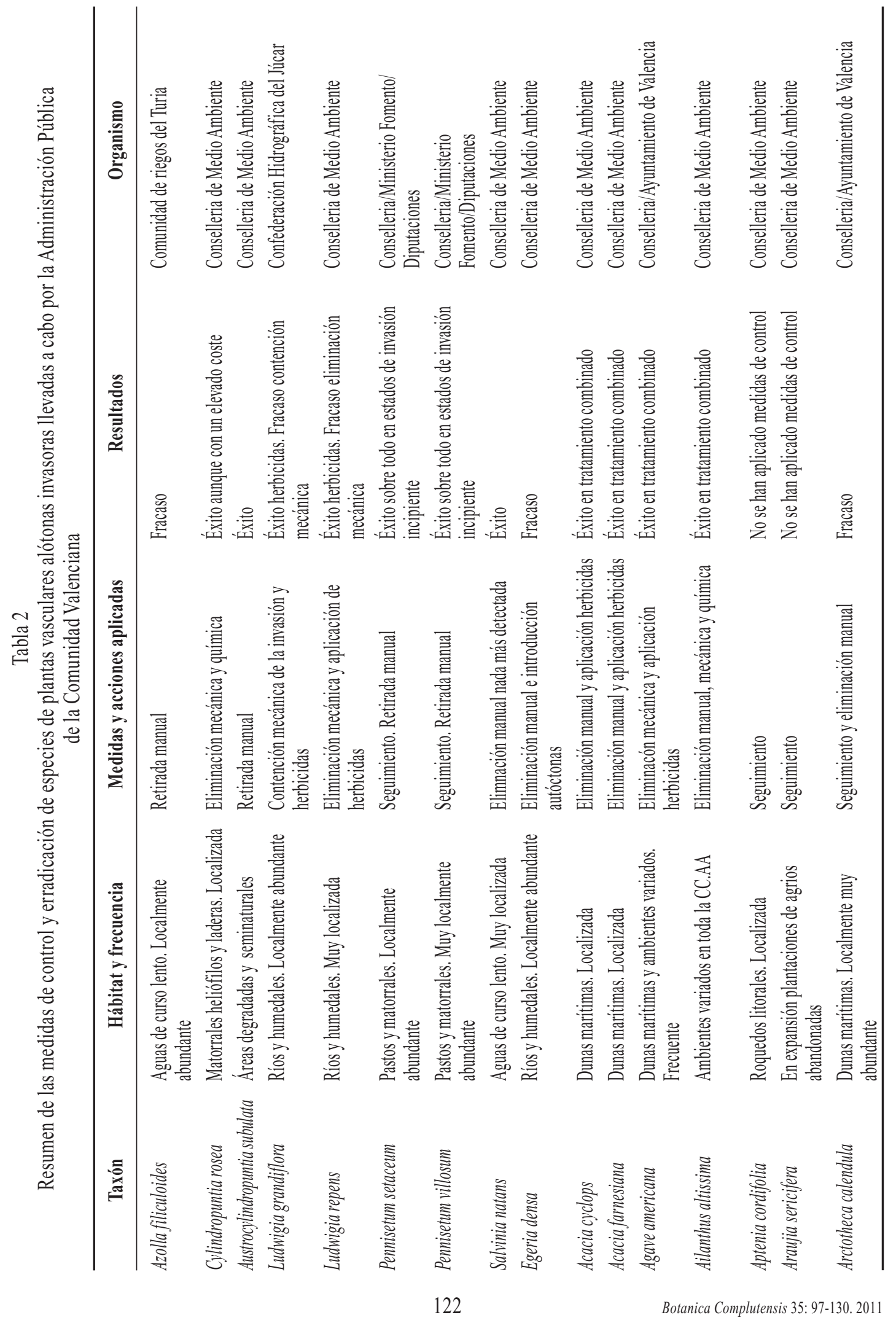




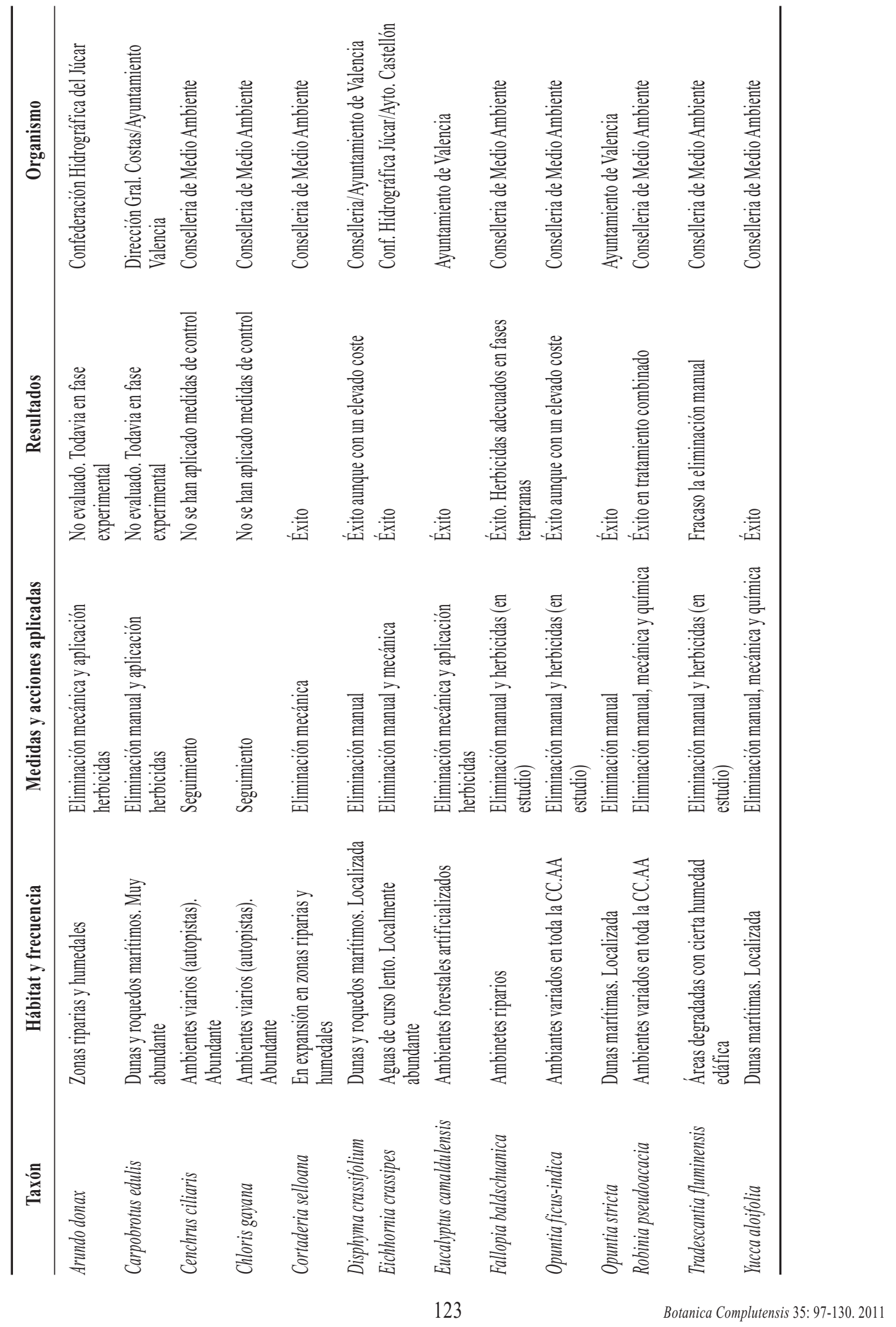


de la Comunidad Valenciana corresponde a especies transformadoras, es decir aquellas que causan alteraciones 0 perturbaciones en el funcionamiento de los ecosistemas. Éstas son: Abutilon theophrasti, Agave americana, Azolla filiculoides, Carpobrotus edulis, Carpobrotus acinaciformis, Araujia sericifera, Cortaderia selloana, Cylindropuntia rosea, Opuntia ficus-indica, Oxalis pes-caprae, Arctotheca calendula, Arundo donax, Cenchrus ciliaris, Chloris gayana, Egeria densa, Ludwigia grandiflora, Lonicera japonica, Eichhornia crassipes, Ailanthus altissima, y Nicotiana glauca. Si analizamos su peligrosidad ambiental, vemos que la mayoría invaden hábitats riparios y fluviales (Azolla filiculoides, Abutilon theophrasti, Cortaderia seIloana, Egeria densa, Ludwigia grandiflora, Eichhornia crassipes), otro buen contingente invade playas, arenales y roquedos marítimos (Agave americana, Carpobrotus edulis, Carpobrotus acinaciformis, Arctotheca calendula, Oxalis pes-caprae, etc.), otras áreas de matorral o de monte mediterráneo degradado (Cylindropuntia rosea), otras lo hacen en zonas próximas a poblaciones donde la vegetación natural se encuentra muy degradada (Opuntia ficusindica), otras invaden las áreas removidas y abiertas en torno a las grandes vías de comunicación (Chloris gayana,
Cenchrus ciliaris) y otras presentan un temperamento más generalista, con una plasticidad ecológica más amplia ( $\mathrm{Ai}$ lanthus altissima, Aranjia sericifera, Nicotiana glauca). No obstante, de esta diferenciación no debe deducirse que cada hábitat con su elenco de especies invasoras es un compartimento estanco, pues son bastantes las especies que pueden invadir más de un tipo de hábitat. Finalmente, hay que señalar que la Comunidad Valenciana es una de las más avanzadas en lo que respecta a la sensibilidad de las Administraciones Públicas tanto hacia el reconocimiento del problema como hacia la adopción de medidas para su resolución. En la Tabla 2 se resumen las actuaciones llevadas a cabo en esta materia, que no sólo atañen a especies transformadoras sino también a algunas invasoras en espacios protegidos, con una clara vocación preventiva. También hay que destacar la adopción de medidas legislativas, aplicadas con un carácter general y dirigidas principalmente a la prevención, concretadas en el Decreto 213/2009, de 20 de noviembre, de la Conselleria de Medio Ambiente, Agua, Urbanismo y Vivienda, por el que se aprueban medidas para el control de especies exóticas invasoras en la Comunitat Valenciana (DOCV 6151 de 24 de noviembre de 2009).

\section{BIBLIOGRAFÍA}

Aguilella, A. 1985. Flora y vegetación de la Sierra del Toro y las Navas de Torrijas (estribaciones sudorientales del Macizo de Javalambre). Tesis doctoral. Universidad de Valencia.

Aguilella, A. 1990. Fragmenta chorologica occidentalia, 26562666. Anales Jard. Bot. Madrid 47(2): 481-482.

Aguilella, A.; Fabregat, C. \& Riera, J. 1993. Notes florístiques i corològiques, 670-703. Collect. Bot. (Barcelona) 22: 141-144.

Alcaraz, F.; Garre, M. \& Sánchez, P. 1985. Catálogo de la flora cormofítica de los sistemas de dunas litorales comprendidos entre Santa Pola y Calblanque (SE de España). Anales Biol. Fac. Biol. Univ. Murcia 6: 79-87.

Alcober, J. A.; Ibars, A.; Mansanet, J.; Mateu, I. \& Puche, M. 1980. Marsilea aegyptiaca Willd. Nueva cita para España. Anales Jard. Bot. Madrid 36: 55-59.

Ballester, G. \& StüBing, G. 1990. Cuadernos de la Naturaleza $n^{0}$ 1: La Sierra del Carrascal de Alcoy. Flora y Vegetación. Alicante.

Ballester, G.; Figuerola, R.; Peris, J. B. \& Stübing, G. 1991. 4.2 Flora y vegetación. En: Estudio multidisciplinar del Parque Natural del Montgó (Alicante): 120-236. Conselleria d'Adminstració Pública \& Agencia del Medi Ambient. Valencia.
BANYULS B. \& SOLER, J. X. 2000. El paisatge vegetal de Teulada (la Marina Alta). Asociació Cultural Amics de Tuelada. Ajuntament de Teulada. Alicante.

Barnades, M. 1785. Viaje desde Madrid a Aranjuez, Albacete, Tobarra, Cieza, Murcia, Cartagena, Guardamar, por la Marina y la Gola, a Alicante, a Mariola por Jijona, Elche, Orihuela, Murcia, Jumilla, Tobarra y por el mismo camino de Albacete a Madrid: desde primeros de mayo hasta mediados de junio de 1785. Manuscrito. Madrid.

Berthet, P. 1990. Opuntia Miller En: S. Castroviejo et al. (Eds.), Flora iberica 2: 63-70. Real Jardín Botánico, CSIC. Madrid.

Bolòs, O. 1967. Comunidades vegetales de las comarcas próximas al mar situadas entre los ríos Llobregat y Segura. Mem. Real Acad. Ci. Barcelona 38: 3-280.

BoLòs, O. 1975. De vegetatione valentina, II. Anales Inst. Bot. Cavanilles 32(2): 477-488.

Bolòs, O. 1979. De vegetatione valentina, III. Butll. Inst. Catalana Hist. Nat., Secc. Bot. 44: 65-76.

Bolòs, O. \& VIGO, J. 1979. Observacions sobre la flora del Països Catalans. Collect. Bot. (Barcelona) 11: 25-89.

Bolòs, O. \& Vigo, J. 1984-2001. Flora dels Països Catalans. 1-4. Editorial Barcino. Barcelona.

BoRJA, J. 1951. Estudio fitográfico de la Sierra de Corbera (Valencia). Anales Jard. Bot. Madrid 9: 361-483. 
Campos, J. A. \& Herrera, M. 2009. Análisis de la flora alóctona de Bizkaia (País Vasco, España). Lazaroa 30: 7-33.

CAmuñas, E. \& CREsPo, M. B. 1998. Neófitos nuevos o interesantes para la flora alicantina. Acta Bot. Malac. 23:210-214.

Camuñas, E. \& CRespo, M. B. 1999. The genus Hoffmannseggia Cav. (Fabaceae, Caesalpinioideae), new for the Meditearranean flora. Isr. J. Plant. Sci. 47: 283-286.

Carrasco, M. A. 1995. Malvella leprosa (Ortega) Krapov. (Malvaceae), introducida en Alicante (España), primera cita para la Península Ibérica. Anales Jard. Bot. Madrid 53(2): 254-255.

Carretero, J. L. 1979. Solanum eleagnifolium Cav. y Cuscuta campestris Yuncker. Nuevas especies para la flora española. Collect. Bot. (Barcelona) 11: 143-154.

Carretero, J. L. 1981. El género Echinochloa Beauv. en el suroeste de Europa. Anales Jard. Bot. Madrid 38(1): 91-108.

Carretero, J. L. 1982. Algunas plantas interesantes de la zona arrocera valenciana. Anales Jard. Bot. Madrid 39(1): 215-216

Carretero, J. L. 1983a. El género Ammannia L. (Lythraceae) en España. Anales Jard. Bot. Madrid 39(2): 273-277.

Carretero, J. L. 1983b. Chenopodium pumilio R. Br. y Physalis philadelphica Lam. en España. Collect. Bot. (Barcelona) $14: 211-213$.

Carretero, J. L. 1985a. Consideraciones sobre las amarantáceas ibéricas. Anales Jard. Bot. Madrid 41(2): 271-286.

Carretero, J. L. 1985b. Aportaciones a la flora exótica valenciana. Collect. Bot. (Barcelona) 16: 133-136.

Carretero, J. L. 1986. Bowlesia incana Ruiz \& Pavón en España. Anales Jard. Bot. Madrid 43(1): 177.

Carretero, J. L. 1987a. Fragmenta chorologica occidentalia, 670-676. Anales Jard. Bot. Madrid 43(2): 439-440.

Carretero, J. L. 1987b. Paspalum saurae (Parodi) Parodi, una grammínea nueva para Europa. Anales Jard. Bot. Madrid 44: $175-176$.

Carretero, J. L. 1988. Wedelia glauca (Ortega) O. Hoffm. ex Hicken. Anales Jard. Bot. Madrid 45(1): 346-347.

Carretero, J. L. 1989. Eichhornia crassipes en la Comunidad Valenciana. Anales Jard. Bot. Madrid 45(2): 568.

Carretero, J. L. 1990. Macrófitos acuáticos de la provincia de Alicante. Medi Natural 2: 45-56.

Carretero, J. L. 1991. Sobre flora alóctona valenciana. Collect. Bot. (Barcelona) 20: 259-261.

Carretero, J. L. 1992. Fragmenta chorologica occidentalia, 4251-4256. Anales Jard. Bot. Madrid 50(1): 107.

Carretero, J. L. 1997a. Sida spinosa L. y Solanum nitidibaccatum Bitter, nuevas alóctonas de la flora arvense valenciana. Fl. Montib. 5: 61-62.

Carretero, J. L. 1997b. Hydrocotyle verticillata Thunb. (Umbelliferae) en la flora ibérica. Fl. Montib. 5: 63.

Carretero, J. L. \& Aguilella, A. 1995. Flora y vegetación nitrófilas del término municipal de la ciudad de Valencia. Ajuntament de Valencia.

Carretero, J. L. \& Costa, M. 1998. Polygonum pensylvanicum L. (Polygonaceae), naturalized in Spain. Anales Jard. Bot. Madrid 56(2): 369
Carretero, J. L. \& Esteras, F. J. 1983. Algunas Gramíneas de interés corológico para la provincia de Valencia. Collect. Bot. (Barcelona) 14: 215-219.

Carretero, J. L. \& Pastor, V. 1987. Fragmenta chorologica occidentalia, 994-999. Anales Jard. Bot. Madrid 44(1): 161-162.

Carretero, J. L.; Boira, H. \& Pastor, V. 1984. Aportaciones al conocimiento de la flora de la provincia de Valencia. Collect. Bot. (Barcelona) 15: 139-143.

CASASAYAS, T. 1989. La flora al.lòctona de Catalunya. Tesis doctoral. Facultad de Biología. Universidad de Barcelona.

Castroviejo, S. 1987. Notas sobre Atriplex L. ibéricas. Anales Jard. Bot. Madrid 43(2): 474-476.

Castroviejo, S. et al. (Eds.) 1986-2005. Flora iberica 1-8, 10, 14, 21. Real Jardín Botánico, CSIC. Madrid.

Cavanilles, A. J. 1797. Observaciones sobre la Historia Natural, Geografía, Agricultura, Población y Frutos del Reyno de Valencia. Madrid.

CID, R. 1971. Guizotia abyssinica (L.) Cassini. Adventicia nueva para la flora española. Bol. Coc. Castellonense Cult. 47: 36-37.

Cires Rodríguez, E.; Fernández Prieto, J. A. \& Bueno SánCHEZ, A. 2006. Estado actual de las plantas alóctonas e invasoras del principado de Asturias. Libro de resúmenes del $2^{\circ}$ Congreso Nacional sobre Especies Exóticas Invasoras. GEIB. León.

Cirujano, S. 1990. Tamarix L. En: S. Castroviejo et al. (Eds.), Flora iberica 2: 437-445. Real Jardín Botánico, CSIC. Madrid.

Cirujano, S.; Medina, L.; StüBing, G. \& Peris, J. B. 1995. Egeria densa Planchon (Hydrocharitaceae), naturalized in Spain and Ludwigia natans Elliot (Onagraceae), a xenophyte new to European Flora. Anales Jard. Bot. Madrid 53(1): $140-141$.

Clary, J. 2008. Rainfall seasonality determines annual/perennial grass balance in vegetation of Mediterranean Iberia. Plant Ecol. 195(1): 13-20.

Colmeiro, M. 1886. Enumeración y revisión de las plantas de la Península Hispano-Lusitana e Islas Baleares. Vol. II. Madrid.

Colmeiro, M. 1888. Enumeración y revisión de las plantas de la Península Hispano-Lusitana e Islas Baleares. Vol. IV. Madrid.

Conca, A.; Oltra, J. E. \& Serra, L. 2002. Proboscidea louisianica (Mill.) Thell. (Martyniaceae), nueva para la Comunidad Valenciana. Fl. Montib. 22: 10-11.

Crespo, M. B. 1989a. Estudio sobre la flora y vegetación del término municipal de Burjasot. Memoria de Licenciatura. Facultad de Biología. Universidad de Valencia.

Crespo, M. B. 1989b. Contribución al estudio florístico, fitosociológico y fitogeográfico de la Sierra Calderona (Valencia-Castellón). Tesis doctoral. Universidad de Valencia.

Crespo, M. B.; García, R. \& Manso, M. L. 1988. Noves dades corològiques d'Eclipta prostrata (L.) L. al País Valencià. Collect. Bot. (Barcelona) 17(2): 310-311. 
Crespo, M. B.; Manso, M .L. \& Mateo, G. 1990. Pennisetum setaceum (Poaceae), especie nueva para el continente europeo. Anales Jard. Bot. Madrid 47(1): 260.

Crespo, M. B.; Camuñas, E. \& Cristóbal, J. C. 2007. Precisiones corológicas y taxonómicas sobre la flora de Alicante. Fl. Montib. 36: 52-64.

Cristóbal, J. C.; Camuñas, E. \& Crespo, M. B. 1998. Chrysanthemoides monilifera (L.) Norl. (Asteraceae) alóctona prácticamente nueva para la flora ibérica. Anales Jard. Bot. Madrid 56(2): 390-391.

Cuchillo, J. \& Gimeno, J. 2005. Flora fontina. Estudio de la flora vascular de la Font de Figuera y terrenos colindantes. Editorial Moliner. Burjassot, Valencia.

Cuchillo, J. \& Gimeno, J. 2006. De flora fontina: aportación al estudio de la flora vascular del suroeste de la provincia de Valencia. Fl. Montib. 32: 8-14.

Dana, E. D.; Sobrino, E. \& Sanz Elorza, M. 2003. Plantas invasoras en España: un nuevo problema en las estrategias de conservación. En: A. Bañares, G. Blanca, J. Güemes, J. C. Moreno \& S. Ortiz (Eds.), Atlas y Libro Rojo de la Flora Vascular Amenazada de España: 1009-1027. Dirección General de Conservación de la Naturaleza. Ministerio de Medio Ambiente. Madrid.

De La Torre, A. 1988. Flora, vegetación y suelos de la Sierra del Maigmó (Alicante). Caja de Ahorros Provincial de Alicante.

De La Torre, A. 1991. Vegetación y suelos en el alto Vinalopó (Alicante). Tesis doctoral. Universidad de Murcia.

De La TORRE, F. 2003. Las plantas invasoras en Asturias. Naturalia Cantabricae 2: 33-43.

De La Torre, A.; Alonso, M. A. \& Viciedo, M. 1996. Adiciones al catálogo de la flora vascular de Alicante (SE de España). Lazaroa 16: 197-200.

Domingues, J. \& Freitas, H. 2001. The exotic and invasive flora of Portugal. Bot. Complut. 25: 317-327.

Donat, M. P. 1988. Flora del Macizo del Montgó (Marina Alta). Memoria de licenciatura. Universidad de Valencia.

FaBREGAT, C. 2002. La colección histórica del Dr. Abelardo Rigual en el herbario ABH: revisión nomenclatural y estudio crítico. Institud d'Estudis Ilerdencs. Lérida.

Fabregat, C.; Rigual, A. \& CRespo, M. B. 2003. Saharanthus ifniensis (Caball.) M.B. Crespo \& Lledó (Plumbaginaceae), novedad para la flora europea. Acta Bot. Malac. 28: 193-195.

FernáNDEZ Quintanilla, C. 2009. ¿Malas hierbas o plantas adventicias? Bol. Soc. Esp. Malherbología 59: 5.

Ferrer, P. P. \& Laguna, E. 2009a. Primeras citas de una caña naturalizada en Valencia. Quercus 278: 46.

Ferrer, P. P. \& Laguna, E. 2009b. Sobre Ludwigia hyssopifolia (G. Don) Exell (Onagraceae) como integrante de la flora subespontánea valenciana. Acta Bot. Malac. 34: 228-230.

FRAGA, P. 2009. Jardinería mediterránea sin especies invasoras. Manuales Técnicos 1. Conselleria de Medio Ambiente, Agua, Urbanismo y Vivienda. Generalitat Valenciana. Valencia.
GANDOGER, M. 1917. Catalogue des plantes récoltées en Espagne et en Portugal pendant mes voyages de 1894 á 1912. París.

Gómez Ortega, C. 1784. Continuación de la flora española, o Historia de las plantas que escribia D. Joseph Quer, 5-6. Madrid.

GonZÁLEZ VÁzquez, E. 1928. Un chopo español del subgénero Turunga (Populus ilicitanus Dode). España Forestal 149: 26-30.

Guara, M.; Ferrer, P. P.; Ciurana, M. J. \& Herrero-BorgoNón, J. J. 2004. Flora alóctona adventicia o naturalizada en la Comunidad Valenciana e Islas Baleares. Fl. Montib. 27: 15-22.

Guara, M.; Ferrer, P. P. \& Olivares, A. 2003. Heteranthera limosa (Sw.) Willd., neófito para la flora valenciana. Fl. Montib. 25: 52-55.

GuilLot, D. 2001. Apuntes sobre algunos neófitos de la flora valenciana. Fl. Montib. 18: 19-21.

GuilLot, D. 2003a. Apuntes corológicos sobre neófitos de la flora valenciana. Fl. Montib. 23: 13-17.

GuiLLOT, D. 2003b. Acerca de cuatro taxones del género Kalanchoe Adanson en la Comunidad Valenciana. Blancoana 20. 47-49.

GuilLOT, D. 2003c. Sobre la presencia de 17 taxones de la familia Cactaceae en la Comunidad Valenciana. Fl. Montib. 24: 6-13.

GuilLot, D. 2006a. Nopalea dejecta Salm-Dyck, un nuevo taxón alóctono para la flora española. Bouteloua 1: 71-72.

GuiLLOT, D. 2006b. Ipomoea nil (L.) Roth e I. hederacea (L.) Jacquin, dos especies invasoras nuevas para la flora valenciana. Acta Bot. Malac. 31: 153-156.

Guillot, D. \& Roselló, J. A. 2004. Lavandula x cavanillesii, un híbrido nuevo en la flora alóctona valenciana. Flora Montiberica 28: 77-79.

Guillot, D. \& Roselló, J. A. 2005. Kalanchoe x hybrida Jacobs., un nuevo taxón invasor en la Comunidad Valenciana. Lagascalia 25: 176-177.

Guillot, D. \& Roselló, J. A. 2006. Un nuevo híbrido dentro del género Lavandula L., Lavandula x glaucescens D. Guillot \& Roselló (L. dentata var. candicans x L. angustifolia Mill.). Bouteloua 1: 61-62.

Guillot, D. \& Van Der Meer, P. 2001. Siete taxones nuevos del género Opuntia Mill. en la provincia de Valencia: aspectos históricos, ecológicos y reproductivos. Fl. Montib. 19: 37-44.

Guillot, D. \& Van Der Meer, P. 2003. Las familias Agavaceae y Aloaceae en la Comunidad Valenciana. Fl. Montib. 23: 29-43.

Guillot, D. \& Van Der MeER, P. 2004. Algunas citas de neófitos en la Comunidad Valenciana. Fl. Montib. 27: 5-7.

Guillot, D. \& Van Der Meer, P. 2005a. Nuevos datos de las familias Agavaceae y Aloaceae en la costa mediterránea de la Península Ibérica. Fl. Montib. 30: 3-8.

Guillot, D. \& Van Der MeER, P. 2005b. Agave segurae D. Guillot \& Van der Meer, un taxon nuevo dentro del grupo Americanae, naturalizado en la Comunidad Valenciana. Fl. Montib. 30: 30-33. 
Guillot, D. \& Van Der MeER, P. 2006a. Algunos taxones nuevos del género Opuntia Mill. en la Comunidad Valenciana. Fl. Montib. 32: 39-50.

Guillot, D. \& Van Der Meer, P. 2006b. Tres taxones invasores pertenecientes al género Opuntia Mill. Bouteloua 1: 52-54.

Guillot, D. \& Van Der Meer, P. 2006c. Opuntia tomentosa Salm-Dyck un nuevo taxón invasor en la Península Ibérica. Lagascalia 27: 177-179.

Guillot, D. \& Van Der Meer, P. 2008a. El género Agave L. en la flora alóctona valenciana. Monografías Bouteloua 3: $1-94$.

Guillot, D. \& Van Der Meer, P. 2008b. Algunas citas nuevas del género Agave L. para la flora alóctona española. Bouteloua 4: 23-31.

Guillot, D. \& Van Der Meer, P. 2009. Agave desmetiana Jacobi, un nuevo taxon alóctono para la flora europea. Acta Bot. Malac. 34: 251-254.

Guillot, D.; Laguna, E. \& Roselló, J. A. 2008a. La familia Crassulaceae en la flora alóctona valenciana. Monografías Boteloua 4: 1-106.

Guillot, D.; Laguna, E. \& Roselló, J. A. 2008b. Flora alóctona valenciana: familia Cactaceae. Monografías Boteloua 5: $1-148$.

Guillot, D.; Laguna, E. \& Roselló, J. A. 2008c. La familia Aloaceae en la flora alóctona valenciana. Monografías Boteloua 6: 1-58.

Guillot, D.; Laguna, E. \& Roselló, J. A. 2008d. Flora alóctona suculenta valenciana: Aizoaceae y Portulacaceae. Monografias de la revista Boteloua 7: 1-68.

HerRero-Borgoñon, J. J. 2003. Dos Cotoneaster (Rosaceae) de uso ornamental naturalizados en Valencia. Fl. Montib. 24: $3-5$.

HerRero-Borgoñon, J. J. 2007. Dos mimosoideas (Leguminosae) nuevas para la flora castellonense. Fl. Montib. 37: 2628.

HerRero-Borgoñon, J. J. 2008. Azolla filiculoides Lam. en el este ibérico. Acta Bot. Malac. 33: 354-356.

Herrero-Borgoñon, J. J. \& Crespo, M. B. 1998. Fragmenta chorologica occidentalia, 6679-6688. Anales Jard. Bot. Madrid 56(1): 147-148.

herrero-Borgoñon, J. J.; Cristóbal, J. C. \& Crespo, M. B. 1995. Pennisetum clandestinum Hochst. ex Chiov. (Poaceae), an african grass in Europe. Isr. J. Plant Sci. 43: 159-162.

Juan, A.; Serra, L.; Cristóbal, J. C.; Barber, A. \& Crespo, M. B. 1996. Notas sobre plantas alicantinas. Bot. Complut. 21: $59-70$.

LAGUNA, E. 2000a. Solanum rostratum y Asclepias physocarpa, dos nuevos neófitos para la flora valenciana. Fl. Montib. 14: 35-37.

LaGunA, E. 2000b. Del nombre botánico de algunos grupos de especies cultivadas, plantadas 0 asilvestradas en el oriente ibérico. II: Hedera, Pinus, Plectranthus. Fl. Montib. 15-21-30.

LaGuNA, E. 2006c. Las especies cultivadas y asilvestradas de grandes palmeras datileras en tierras valencianas. Bouteloua 1: 6-12.
Laguna, E. \& Mateo, G. 2001. Observaciones sobre la flora alóctona valenciana. Fl. Montib. 18: 40-44.

Laguna, E. \& SÁnchez De Lorenzo-Cáceres, J. M. 2009. Dodanea viscosa Jacq. (Sapindaceae), novedad florística para la Península Ibérica. Fl. Montib. 23: 3-7.

Laguna, E.; Crespo, M. B.; López Udias, S.; Fabregat, C.; SeRRA, L.; Herrero-BorgoÑón, J. J.; CARRetero, J. L.; Agullella, A. \& Figuerola, R. 1998. Flora rara, endémica o amenazada de la Comunidad Valenciana. Conselleria de Medio Ambiente. Generalitat Valenciana. Valencia.

Laguna, E.; Ferrer, P. P. \& Currás, R. 2010. Sobre la presencia de tres nuevas alóctonas asilvestradas para la flora peninsular ibérica. Studia Botanica (en prensa).

LÓPEZ, M.; IZqUiERDO, I.; MARTíN, J. L. \& RODRíGuEZ, J. L. 2003. Algunos datos sobre las especies exóticas de Canarias: hipótesis sobre la preferencia de hábitat. En VV. AA. (Eds.), Contribuciones al conocimiento de las especies exóticas en España. I Congreso Nacional sobre Especies Exóticas Invasoras: 94-95- GEI. León.

LOSA, T. M. 1948. Algo sobre especies españolas del género «Euphorbia» L. Anales Jard. Bot. Madrid 7: 357-431.

Loscos, F. \& PARDO, J. 1866-1867. Serie imperfecta de las plantas aragonesas espontáneas. Alcañiz, Teruel.

Mansanet, J. \& Aguilella, A. 1984. Notas florísticas valencianas, VI. Lazaroa 6: 287-289.

Martínez MartíneZ, M. 1934. Aportaciones a la flora española, plantas de Alicante. Mem. Real Soc. Esp. Hist. Nat. 14: 405-480.

Martínez Fort, J. \& Donat, M. P. 2006. Crassula alata (Viv.) Berger subsp. alata; primera cita para la Península Ibérica. Fl. Montib. 33: 41-44.

Mateo, G. 2000. Contribuciones a la flora del Sistema Ibérico, XIII. Fl. Montib. 14: 14-16.

Mateo, G. 2008. De flora valentina, IX. Fl. Montib. 39: 33-36.

Mateo, G. \& CRespo, M. B. 1990. Claves para la flora valenciana, del Cènia al Segura. Valencia.

Mateo, G. \& Crespo, M. B. 1995. Flora abreviada de la Comunidad Valenciana. Alicante.

Mateo, G. \& Crespo, M. B. 1998. Manual para la determinación de la flora valenciana. $1^{\text {a }}$ edición. Monografías $F$. Montib. 3. Valencia.

Mateo, G. \& CResPo, M. B. 2001. Manual para la determinación de la flora valenciana. $2^{\mathrm{a}}$ edición. Editorial Moliner. Valencia.

Mateo, G. \& CRespo, M. B. 2003. Manual para la determinación de la flora valenciana. $3^{\text {a }}$ edición. Monografías Fl. Montib. 4. Valencia.

Mateo, G. \& CRespo, M. B. 2009. Manual para la determinación de la flora valenciana. $4^{\mathrm{a}}$ edición. Monografías $\mathrm{Fl}$. Montib. 5. Valencia.

Mateo, G. \& Figuerola, R. 1986. De Flora Valentina, I. Collect. Bot. (Barcelona) 16(2): 377-382.

Mateo, G. \& Marín, F. 1996. De Flora Valentina, V. Fl. Montib. 4: 26-28.

Mateo, G. \& Mercadal, N. E. 1996. Aportaciones a la flora aragonesa, I. Fl. Montib. 3: 47-52. 
Mateo, G. \& Nebot, J. R. 1988. Fragmenta chorologica occidentalia, 1494-1516. Anales Jard. Bot. Madrid 45(1): 307-309.

Mateo, G. \& SerRa, L. 1991. Fragmenta chorologica occidentalia, 3733-3754. Anales Jard. Bot. Madrid 49(1): 133-134.

Mateo, G.; Crespo, M. B. \& Nebot, J. R. 1989. Notulae Chorologicae Valentinae, I. Folia Bot. Misc. 6: 87-92.

Mateo, G.; García Navarro, E. \& Serra, L. 1992. Fragmenta chorologica occidentalia, 4262-4279. Anales Jard. Bot. Madrid 50(1): 106-107.

Mcgeoch, M. A.; Butchart, S. H.; Spear, D.; Marais, E.; Kleynhans, E. J.; Symes, A.; Chanson, J. \& Hoffmann, M. 2010. Global indicators of biological invasion: species number, biodiversity impact and policy responses. Diversity and Distributions 16: 95-108.

Mira, F. 1906. Las dunas de Guardamar. Mem. Real Soc. Esp. Hist. Nat. 4: 57-77.

Molero, J. \& Rovira, M. 1981. De Flora Dianicae. Anales Jard. Bot. Madrid 38(1): 303-305.

Moragues, E. \& Rita, J. 2005. Els vegetals introduits a les illes Balears. Documents Tècnics de Conservació 11. Govern de les Illes Balears. Palma de Mallorca.

MoRin, N. R. (Ed.) 1993-2007. Flora of North America. Oxford University Press. Nueva York.

Nевот, J. R. 1986. Aportació al coneixement de la flora vascular de la Serra de Benicadell (La Vall d'Albaida-El Comtat). Tesis de licenciatura. Inédita. Universidad de Valencia.

Nebot, J. R. \& Mateo, G. 1993. Fragmenta chorologica occidentalia, 4666-4682. Anales Jard. Bot. Madrid 51(1): 135-136.

Nebot, J. R.; De La Torre, A.; Mateo, G. \& Alcaraz, F. 1990. Materiales para la actualización del catálogo florístico de la provincia de Alicante. Anales Biol., Fac. Biol., Univ. Murcia 16: 99-129.

Nogueira, I. 1990. Phytolacca L. En: S. Castroviejo et al. (Eds.), Flora iberica 2: 53-56. Real Jardín Botánico, CSIC. Madrid.

Nogueira, I \& Paiva, J. 1993a. Kosteletzya C. Presl. En: S. Castroviejo et al. (Eds.), Flora iberica 3: 193-196. Real Jardín Botánico, CSIC. Madrid.

Nogueira, I \& Paiva, J. 1993b. Malope L. En: S. Castroviejo et al. (Eds.), Flora iberica 3: 199-201. Real Jardín Botánico, CSIC. Madrid.

PAU, C. 1899. La Oenothera speciosa en España. Actas Soc. Esp. Hist. Nat. 28: 212-213.

Pau, C. 1903. La Oxalis cernua Thumb. Bol. Soc. Aragonesa Ci. Nat. 2: 44.

PAU, C. 1905. Materiales para la flora valenciana según los datos que suministra Cavanilles en su obra Observaciones sobre el Reino de Valencia en los años 1795 (el tomo I) y 1797 (el II). Tipografía Moderna. Valencia.

Peña, C.; Sebastián, A. \& Laguna, E. 2003. Cyperus papyrus en la Albufera de Valencia. Fl. Montib. 23: 44-45.

Pérez BAdíA, M. R. 1996. Aportaciones al conocimiento de la flora diánica. Lazaroa 16: 201-204.
PÉREZ BADÍA, M. R. 1997. Flora vascular y vegetación de la comarca de la Marina Alta (Alicante). Instituto de cultura "Juan Gil-Albert". Diputación Provincial de Alicante.

Pérez Badía, M. R.; Molina, J. A. \& Soriano, P. 1995. Eleocharis bonariensis Nees en el suroccidente europeo: distribución y diferencias frente a Eleocharis acicularis (L.) Roemer \& Schultes. Lazaroa 15: 145-150.

Pérez Badía, M. R.; De La Torre, A.; Serra, L.; \& Crespo, M. B. 1994. Notas corológicas sobre plantas alicantinas. Fontqueria 40: 25-29.

Perez Dacosta, J. M. 2004. Aportaciones a la flora de la comarca de La Plana (Castellón). Fl. Montib. 24: 12-18.

PERIS, J. B. 1983. Contribución al estudio florístico y fitosociológico de las sierras de Boquerón y Palomera. Tesis doctoral. Universidad de Valencia.

Peris, J. B.; STÜBING, G. \& GonZÁLez, E. 1984. Ipomoea stolonifera, un neófito litoral-psamófilo nuevo para la Flora Ibérica. Anales Jard. Bot. Madrid 40(2): 467-468.

PORTA, P. 1892. Vegetabilia in itinere iberico austro-meridionali lecta. Atti Imp. Regia Accad. Rovereto 9: 104-177.

PUERTA, G. 1876. Tratado práctico de determinación de plantas. Madrid.

Pyšek, P.; Richardson, D. M.; Rejmánek, M.; Webster, G. L.; Williamson, M. \& Kirschner, J. 2004. Alien plants in checklists and floras: towards better communication between taxonomists and ecologists. Taxon 53(1): 131-143.

PyšEK, P.; LAMBdon, P. W.; Arianoutsou, M.; Kühn, I.; Pino, J. \& WinTER, M. 2009. Alien vascular plants of Europe. En E. Hulme, W. Nentwig, P. Pyšek \& M. Vilà (Eds.), Handbook of alien species in Europe: 43-61. Springer Science.

RAUNKJAER. O. 1934. The life forms of plants and stastistical plant geography. Clarendon Press. Reino Unido.

Richardson. D. M.; Pysek. P.; RejmÁnek, M.; BarbouR, M. G.; PANeTtA, F. D. \& WeST, C. J. 2000. Naturalization and invasion of alien plants: concepts and definitions. Diversity and Distribution 6: 93-107.

RIERA, J. 1992. Aproximació al coneixement florístic de la Serra de Pina. Universidad de Valencia. Jardí Botanic de Valencia. Memoria de Licenciatura.

RiguaL, A. 1972. Flora y vegetación de la provincia de Alicante. Diputación Provincial de Alicante. Reeditada y ampliada en 1984.

RiverA, D. \& Ruiz, J. B. 1987. Argania spinosa (L.) Skeels (Sapotaceae) subespontánea en la Península Ibérica. Anales Jard. Bot. Madrid 44(1): 173.

Rivera, D.; Obón De Castro, C.; Ríos, S.; Selma, C.; MénDEZ, F.; Verde, A. \& CANO, F. 1998. Las variedades tradiciones de frutales de la cuenca del río Segura. Catálogo etnobotánico. Cítricos, frutos carnosos y vides. DM Librero Editor. Murcia.

Ríos, S.; Robledo, A. \& Alcaraz, F. 1992. Notas sobre la flora alóctona del sureste ibérico, I. Anales Biol. 18: 95-102. 
Ríos, S.; Martínez, F.; Martínez-Francés, V. \& Moyti, N. 2008. Algunas citas de interés para la flora valenciana (norte de Alicante). Fl. Montib. 40: 30-33.

Rocha Afonso, M. L. 1990. Platanus L. En: S. Castroviejo et al. (Eds.), Flora iberica 2: 3-5. Real Jardín Botánico, CSIC. Madrid.

Romero BuJÁn, M. I. 2007. Flora exótica de Galicia (noroeste ibérico). Bot. Complut. 31: 113-125.

Roselló, R. 1994. Catálogo florístico y vegetación de la comarca natural del Alto Mijares. Diputaciò de Castellò.

Roselló, R. \& Peris, J. B. 1983. Algunos neófitos de la provincia de Castellón. Fontqueria 28: 53-56.

RouY, G. 1883. Excursions botaniques en Espagne par Orihuela, Murcia, Vélez-Rubio, Hellin, Madrid, Irún (mai 1881juin 1882). Boehm et Fils. Montpellier.

RoYo, F. 2006. Flora i vegetación de les planes i serres litorals compreses entre el riu Ebre i la Serra d'Irta. Tesis doctoral. Universitat de Barcelona.

SAmo, E. 1995. Catálogo floristico de la provincia de Castellón. Diputació de Castelló.

SANchís, E. 1987. Estudio de la flora e introducción al conocimiento de la vegetación de la sierra de Santa María y otras sierras colindantes (Valencia). Tesis doctoral. Universidad de Valencia.

SANCHÍs, E. 1989. Flora y vegetación de la desembocadura del río Segura y sus alrededores. Cuadernos de INICE-Biología II-20: 19-46.

Sanz Elorza, M.; Dana, E. D. \& Sobrino, E. 2004. Atlas de las plantas alóctonas invasoras en España. Dirección General para la Biodiversidad. Ministerio de Medio Ambiente. Madrid.

Sanz Elorza, M.; Dana, E. D. \& Sobrino, E. 2006. Invasibility of an inland area in NE Spain by alien Spain. Acta Oecologica 29: 114-122.

Sanz Elorza, M.; González Bernardo, F. \& Gavilán IgleSIAS, L. P. 2008. La flora alóctona de Castilla y León (España). Bot. Complut. 32: 117-137.

Sanz Elorza, M.; González Bernardo, F. \& Serreta Oliván, A. 2009. La flora alóctona de Aragón (España). Bot. Complut. 33: 69-88.

Sanz Elorza, M.; Mateo, R. G. \& González Bernardo, F. 2009. The historical role of agriculture and gardening in the introduction of alien plants in the western Mediterranean. Plant Ecol. 202: 247-256.

SegarRa, J. G. 2001. Datos sobre la Pteridoflora subespontánea ibérica: Cyrtomium falcatum (Dryopteridaceae) y Nephrolepis cordifolia (Nephrolepideaceae). Nota Pteridológica 49. Acta Bot. Malac. 26: 247-249.

Sennen, F. 1910. Plantes observées autour de Teruel. Bol. Soc. Aragonesa Ci. Nat. 9: 173-184.

Sennen, F. 1911. Note sur la flore de Benicarló, Peñíscola, Sta. Magdalena, etc. Bol. Soc. Aragonesa Ci. Nat. 10: 131-143.

SenNen, F. 1912. Quelques formes nouvelles ou peu connues de la flore de Catalogne, Aragón, Valence. Bol. Soc. Aragonesa Ci. Nat. 11: 177-215.
Sennen, F. 1916. Liste des plantes. Bol. Soc. Aragonesa Ci. Nat. 15: 94-105.

SerRA, L. 1999. La flora de Santa Pola. Ajuntament de Santa Pola. Alicante.

SERRA, L. 2007. Estudio crítico de la flora vascular de la provincia de Alicante: aspectos nomenclaturales, biogeográficos y de conservación. Ruizia 19: 1-1414.

SerRa, L. \& Crespo, M. B. 1998. Adiciones a la flora alicantina, III. Fl. Montib. 9: 20-23.

SerRa, L., Soler, J. X. \& Mateo, G. 1993. Fragmenta chorologica occidentalia, 4683-4697. Anales Jard. Bot. Madrid 51(1): 136-137.

SerRa, L., Pérez Botella, J. \& Izquierdo, J. 2005. El género Periploca L. en la Comunidad Valenciana. Fl. Montib. 31:39-43.

SierRa RÀFols, E. 1979. Algunes espécies adventícies i naturalitzades. Collect. Bot. (Barcelona) 11: 297-300.

Sobrino, E.; Sanz Elorza, M.; Dana, E.D. \& González MoRENO, A. 2002. Invasibility of a coastal strip in NE Spain by alien plants. J. Veg. Sci. 13: 585-594.

Solanas, J. L. 1996. Flora, vegetació i fitogeografía de la Marina Baixa, Alacant. Tesis doctoral. Universidad de Alicante.

Solanas, J. L. \& Mateo, G. 1991. Plantes de la Serra de la Serrella (El Comtat-La Marina Baixa). Butll. Inst. Catalana Hist. Nat., Secc. Bot. 59: 75-80.

Solanas, J. L.; De La Torre, A. \& Crespo, M. B. 1993. Fragmenta chorologica occidentalia, 4632-4658. Anales Jard. Bot. Madrid 51(1): 133-134.

Soler, J. X.; Rochet, B.; Mateo, G. \& SerRa, L. 1995. Fragmenta chorologica occidentalia, 5479-5509. Anales Jard. Bot. Madrid 53(1): 113-114.

Soler, J. X.; Serra, L.; Mateo, G. \& Crespo, M. B. 1995. Adiciones a la flora alicantina. Fl. Montib. 1: 23-28.

Tirado, J.; Villaescusa, C. \& Aguilella, A. 1994. Fragmenta chorologica occidentalia, 4921-4961. Anales Jard. Bot. Madrid 51(2): 293-295.

ToRrente, P. \& EgEA, J. M. 1984. Aportación al conocimiento de los líquenes epífitos del SE de Espana: Líquenes con Trentepohlia. Folia Bot. Misc. 4: 81-89.

Tutin, T. G.; Heywood, V. H.; Burges, N. A.; Moore, D. M.; VAlentine, D. H. \& WebB, D. A. (Eds.). 1964-1980. Flora Europaea 1-5. Cambridge University Press, Cam-bridge.

Urios, V.; Donat, M. P. \& Viñals, M. J. 1993. La Marjal de Pego-Oliva. El Medi Natural de la Marjal de Pego-Oliva i el seu entorn. Institut d'Estudis de la Marina Alta. Pedreguer, Alicante.

VAYREDA, E. 1901: Notas geográfico-botánicas. Anales Soc. Esp. Hist. Nat. 29(3): 363-384.

VAZqueZ, J. R. 2003. Flora de Navajas, Gaibiel y el pantano de Regajo. Trabajo de Investigación. Facultad de Farmacia. Universidad de Valencia.

VerLoove, F. 2006. Exotic grasses running wild: Megathyrsus maximus var. pubiglumis (Poaceae, Paniceae) new to Spain. Bouteloua 1: 55-60.

Verloove, F. \& SÁnchez Gullón, E. 2008. New records of interesting xenophytes in the Iberian Peninsula. Acta Bot. Malac. 33: 147-167. 
Vicedo, M. \& De La Torre, A. 1997. La Sierra de Crevillente: flora y vegetación. Instituto de Cultura Juan Gil-Albert. Alicante.

Vicioso, C. 1915. Una visita a los montes de Bicorp (Valencia). Bol. Soc. Esp. Hist. Nat. 15(1): 90-94.

Vicioso, C. 1916. Plantas de Bicorp (Valencia). Bol. Soc. Esp. Hist. Nat. 16(3): 135-145.

Vicioso, C. 1955. Genisteas españolas, II. Bol Inst. Forest. Invest. Exp. 72: 155-258.
VIG0, J. 1976. Sobre algunas plantas alóctonas. Collect. Bot. (Barcelona) 10: 351-364.

Willкomm, M. 1893. Supplementum Prodromi Florae Hispanicae sive enumeration et description omnium plantarum inde ab anno 1862 usque ad annum 1893 in Hispania detectarum quae innotuerunt auctori locis novis specierum jam notarum. Stuttgart.

Willkomm, M. \& Lange, J. 1861-1880. Prodomus Florae Hispanicae, 1,2 y 3 . Stuttgart. 